\section{LA-8138-MS}

Informal Report
DR. 461

A Finite Element Model for Nonlinear Shells of Revolution

to

$\frac{2}{\frac{0}{9}}$

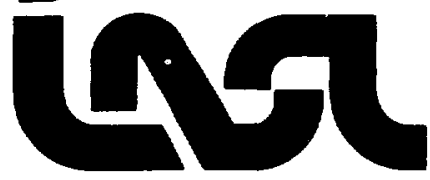




\title{
A Finite Element Model for Nonlinear Shells of Revolution
}

\author{
W. A. Cook
}


TABLE OF CONTENTS

Page

NOMENCL ATURE

vi

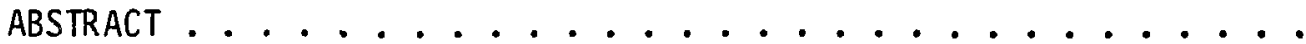

I. INTRODUCTION AND SUMMARY .............

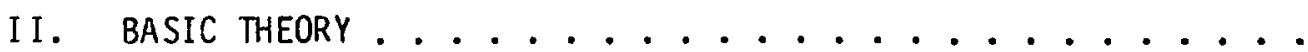

III. NONLINEAR SHELL-OF-REVOLUTION MODEL. . . .......

iv. FINITE ELEMENT APPROXIMATION .............

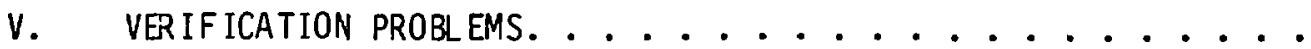

$V I$. CONCLUSIONS AND RECCMMENDATIONS. ...........

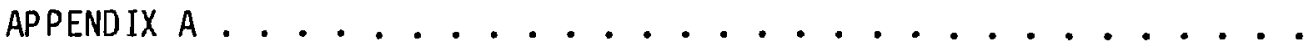

APPENDIX $8 \ldots \ldots \ldots \ldots$

APPENDIX $c \ldots \ldots \ldots \ldots \ldots$

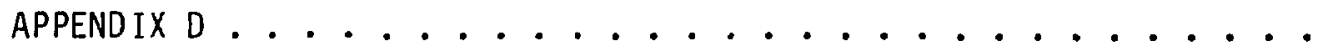

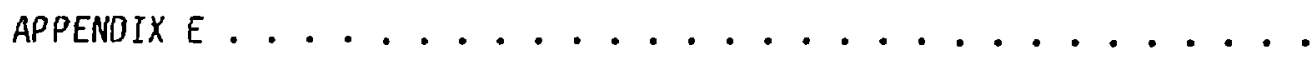

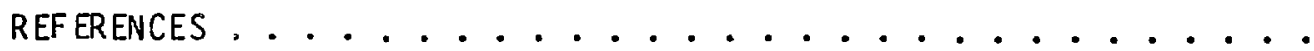




\section{NOMENCLATURE}

- Boundary for shells (ends); also the B matrix is the strain displacement approximation matrix.

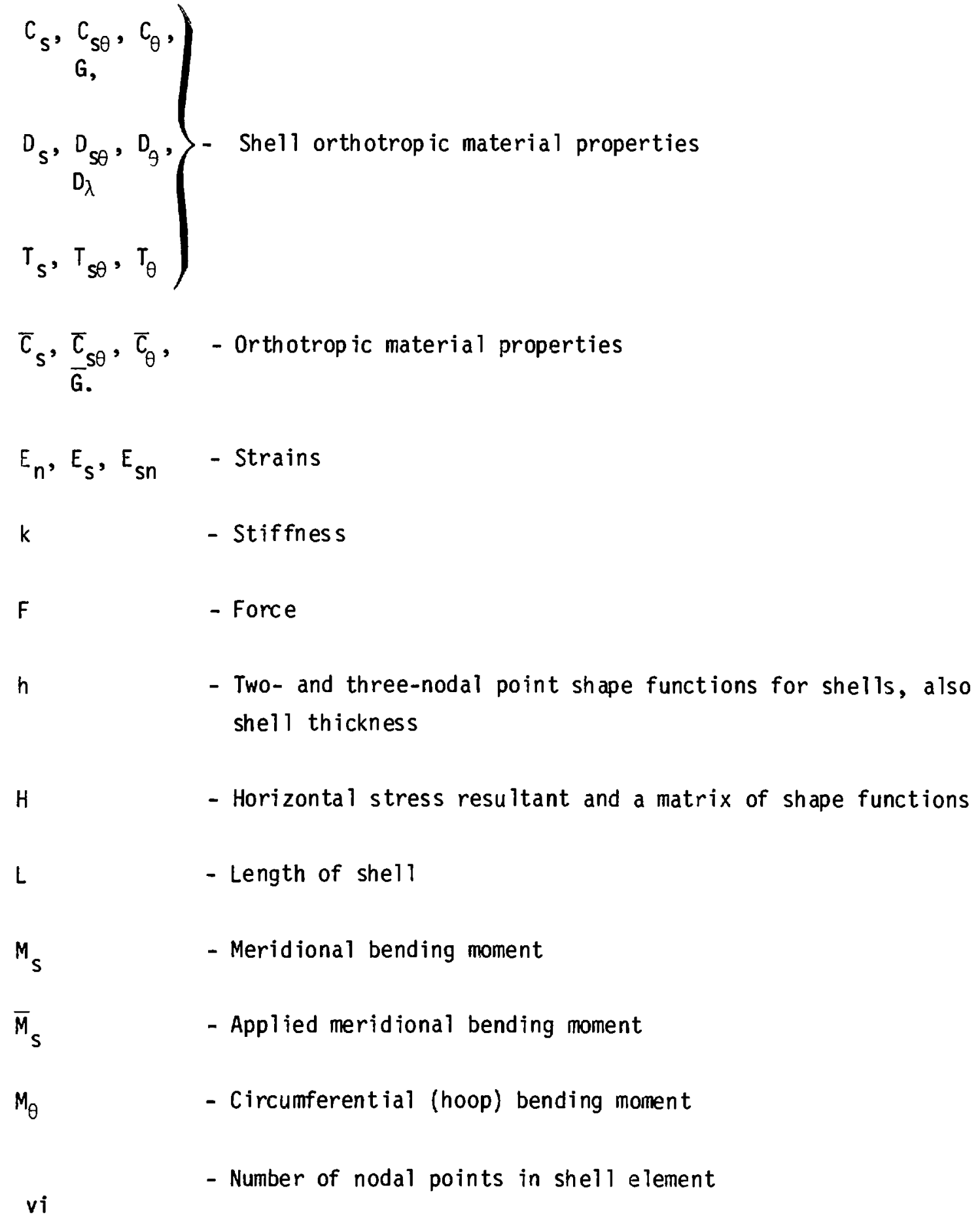




\begin{tabular}{|c|c|}
\hline $\mathrm{N}^{\mathrm{S}}$ & - Meridional stress resultant \\
\hline$N_{\theta}$ & - Circumferential (hoop) stress resultant \\
\hline $\mathrm{p}$ & - Pressure on shell \\
\hline Q & - Shear stress resultant \\
\hline $\bar{Q}$ & - Applied shear stress resultant \\
\hline q & - Meridional variable (ds $=q d \xi)$ \\
\hline$R$ & - Radius of curvature \\
\hline $\mathrm{S}$ & - Shear on shell \\
\hline s & - Meridional coordinate \\
\hline$u_{n}$ & - Nomal displacement \\
\hline$u_{r}$ & - Radial displacement \\
\hline$u_{s}$ & - Meridional displacement \\
\hline$u_{2}$ & - Axial displacement \\
\hline V & - Vertical stress resultant \\
\hline$z$ & - Axial coordinate \\
\hline
\end{tabular}

\section{SUBSCRIPTS}

j

Increment (or iteration)

$j+1$ 

conf iguration

\section{GREEK}

$x$

$\beta$

$\gamma$

$\Gamma(\zeta)$

$\delta$

$\Delta$

$\varepsilon_{s}$

$\varepsilon_{\theta}$

$\zeta$

$\theta$

$\kappa_{S}$

$\kappa_{\theta}$

$\xi$

$\phi$
- Thermal coefficient of expansion

- Rotation $\phi-\phi_{0}$

- Shear deformation

- Shear deformation as a function of thickness

- Variational operator

- Incremental operator and temperature increment when used with a

- Meridional membrane strain

- Circumferential (hoop) membrane strain

- Thickness variable

- Circumferential (hoop) coordinate (1 radian)

- Meridional bending strain

- Circumferential (hoof) bending strain

- Normalized meridional coordinate for shell element

- Azimuthal angle for shell configuration 
A FINITE ELEMENT MODEL FOR NONLINEAR SHELLS OF REVOLUTION

by

W. A. Cook

\section{ABSTRACT}

A finite element model for symmetrically loaded shells of revolution is described. The nonlinear geometric effects are accounted for by incrementing loads and iterating for equilibrium. The iteration process also allows for nonlinear materials. The shell model accounts for large strains, large rotations, and shear deformation. Three example problems demonstrate the versatility and accuracy of this model. An axially loaded c.ylinder and an internally pressurized spherical shell have large membrane strains, whereas a cylinder that deforms into a spherical shape has large rotations.

\section{INTRODUUCTION AND SUMMARY}

Nuclear material shipping containers have shells of revolution as basic siructural components (see Fig. 1). Analytically modeling the response of these containers to severe accident impact conditions requires a nonlinear she11-of-revolution model that accounts for both geometric and material nonlinearities. In this paper I present a finite element model for a nonlinear shell of revolution that will account for large displacements, large strains, 1 arge rotations, and non inear materials. 


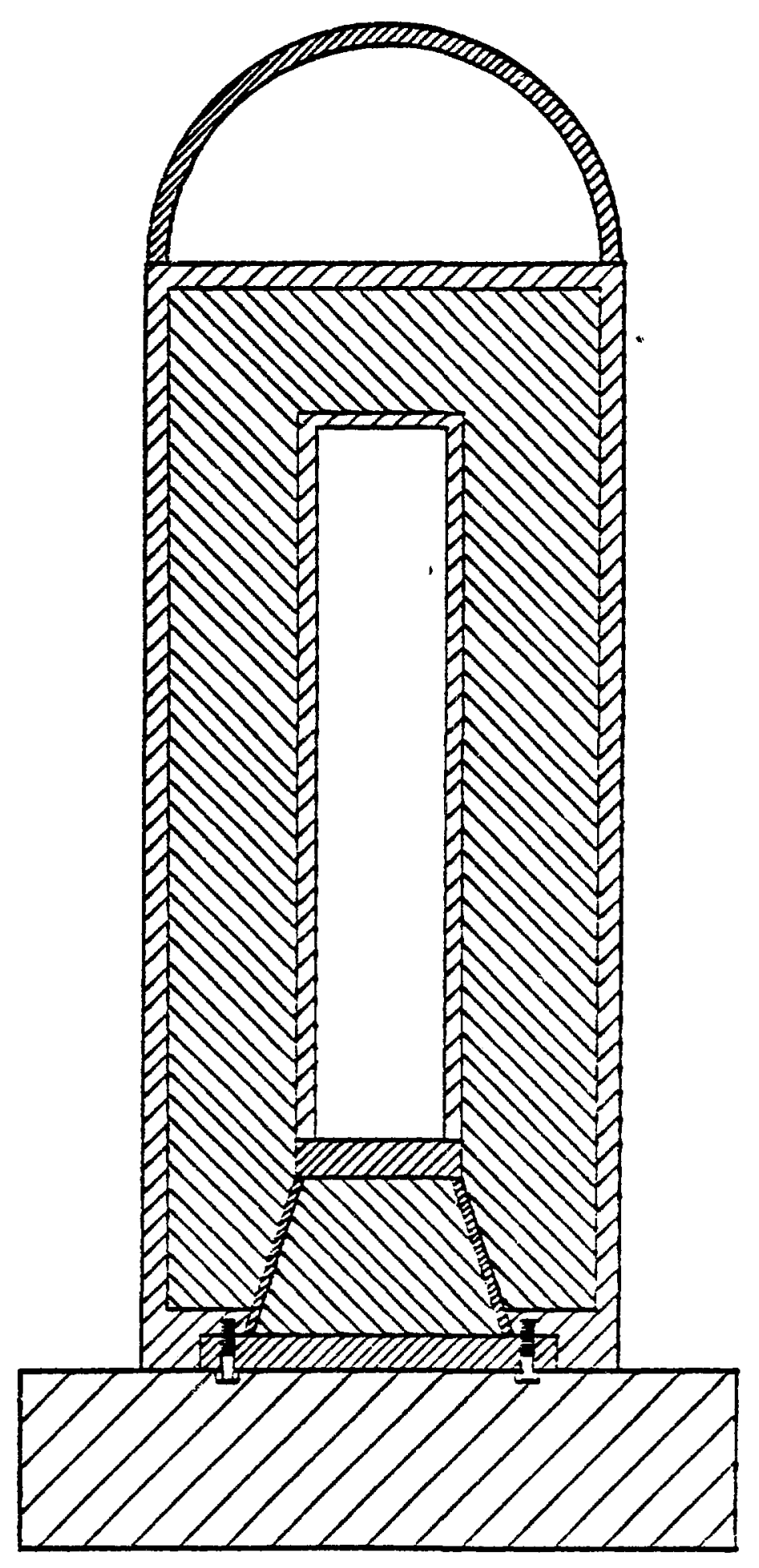

Fig. 1.

A typical nuclear material shipping container. 
The nonlinear shell theory presented by Eric Reissner in Refs. 1 and 2 was used to develop our model and is described in Sec. II. Reissner's approach includes transverse shear deformation and moments turning about the middle surface normal.

This nonlinear shell model is developed using the virtual work principle associated with Reissner's equilibrium equations. First, the virtual work principle is modified for incremental loading; then it is linearized by assuming that the nonlinear portions of the strains are known. By iteration, equilibrium is then approximated for each increment. A benefit of this approach is that this iteration process makes it possible to use nonlinear material properties. The details of this method are presented in Sec. III.

To interface with finite element continulim models with two- and threenodal-point boundaries, our model has both two- and three-nodal-point isoparametric elements. The two-nodal-point element is a conical element, whereas the three-nodal-point element includes curvature terms obtained from a quadratic curve $f$ it of the geometry of the three nodal points. The displacements and meridional rotation are the basic field variables in the elements. For the conical element, these are approximated with linear functions; for the higher order element, these variables are approximated with quadratic functions. The $f$ inite element method as it was used in this incremental/iteration technique is described in Sec. IV.

Several analytical problems were studied using tinis model in the NONSAP computer code. 3,4

- A plate and a cylinder, both with shear loadings, and a portion of a hemisphere with an applied bending moment were analyzed. These loadings were all small, and the model converged quickly to the linear solutions.

- A hemispherical shell was pressurized. The pressure was large enough to cause a normal displacement equal to the original radius of the she 11 .

- An axially loaded cylinder was stretched to double its original length. The last two problems demonstrate the ability of the model to calculate large membrane displacements and nonlinear membrane strains.

- A cylindrical shell was deformed into a spherical shape. The loads required for this problem were calculated from the initial and final configurations and the equilibrium conditions. 
This last exercise demonstrates the ability of the model to be used in analyzing problems with large rotations.

\section{BASIC THEORY}

Figures 2 and 3 show an incremental deformed shell of revolution. From these figures, the equilibrium equations can be derived as (see Appendix A)

$\frac{d\left(r N_{s}\right)}{d s}+r Q \frac{d \phi}{d s}-N_{\theta} \cos \phi+r \bar{S}=0$,

$\frac{d(r Q)}{d s}-r N_{s} \frac{d \phi}{d s}-N_{\theta} \sin \phi+r \bar{p}=0$,

and

$\frac{d\left(r M_{s}\right)}{d s}+r N_{s} \sin \gamma-r Q \cos \gamma-M_{\Theta} \cos \phi+P \sin \phi=0$,

where $\bar{S}=S \cos \gamma-p \sin \gamma$ and $\bar{p}=S \sin \gamma+p \cos \gamma$.

These equations include transverse shear deformation, $\gamma$, and moments turning about the surface normal, $P$. These terms are extensions to the usual thin shell theories and make it possible to model thicker shells.

If we define $q$ and $q_{0}$ such that $d s=q d \xi$ and $d s_{0}=q_{0} d \xi$ (the subscript 0 refers that quantity to the undeformed geometry), then the stress resultants, bending moments, and loads transform as

$N_{s}=\frac{r_{0}}{r} N_{S O}, \quad Q=\frac{r_{0}}{r} Q_{0}, \quad M_{S}=\frac{r_{0}}{r} M_{s o}, \quad N_{Q}=\frac{q_{0}}{q} N_{\theta 0}$,

$P=\frac{q_{0}}{q} p_{0}, \quad M_{\theta}=\frac{q_{0}}{q} M_{\theta 0}, \quad S=\frac{r_{0} q_{0}}{r q} S_{0}, \quad$ and $\quad p=\frac{r_{0} q_{0}}{r q} p_{0}$.

For the meridional bending strain 


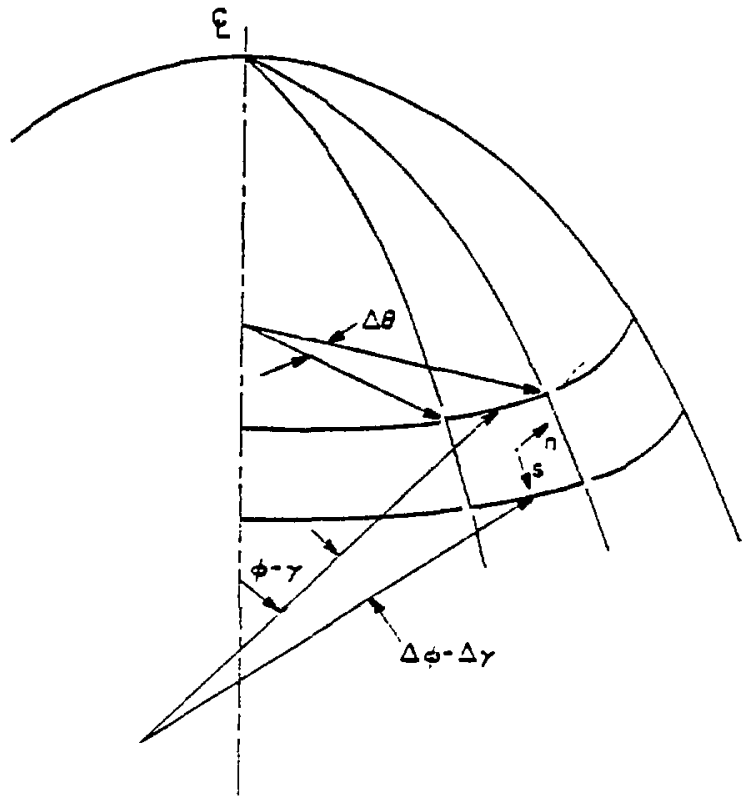

Fig. 2 .

Shel1 of revolution.

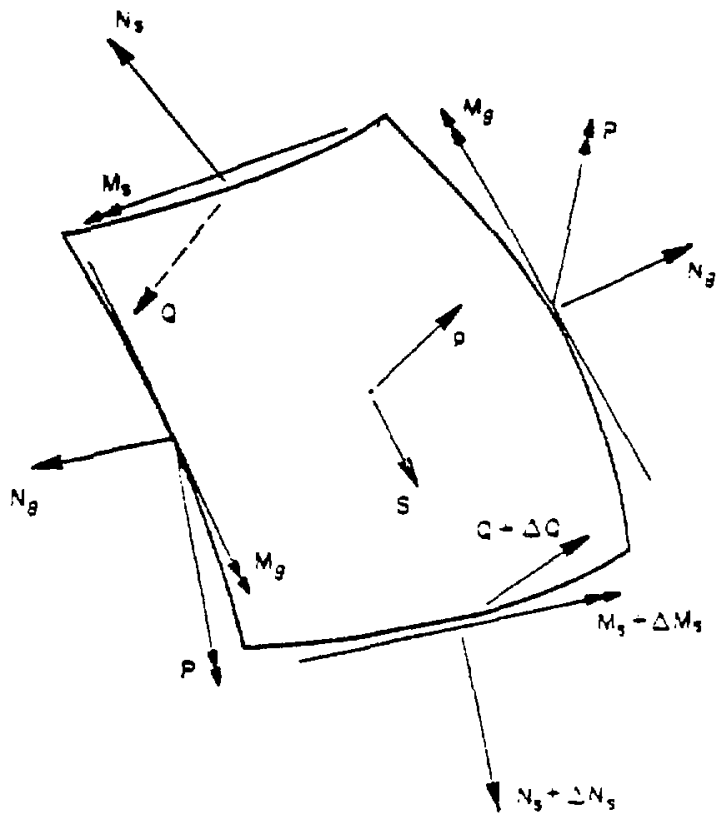

Fig. 3.

Stress resultants, bending moments, and loads for a symmetrically loaded increment of a she1l of revolution. 


$$
\kappa_{s_{0}}=\frac{d \phi}{d s_{0}}-\frac{d \phi_{0}}{d s_{0}}
$$

and because $d s_{0}=R_{0} d \phi_{0}$,

$$
\frac{d \phi}{d s}=\frac{q_{0}}{q}\left(\kappa_{s o}+\frac{1}{R_{0}}\right)
$$

Also, the meridional strain

$$
\varepsilon_{\text {so }}=\frac{q-q_{0}}{q_{0}}
$$

Thus

$$
q / q_{0}=1+\varepsilon_{\text {so }} \text {, }
$$

and the equilibrium equations may be written in terms of undeformed quantities as

$$
\frac{d\left(r_{0} N_{s o}\right)}{d s_{0}}+r_{0} Q_{0}\left(\frac{I}{R_{0}}+k_{s o}\right)-N_{0} \cos \phi+r_{0} \bar{s}_{0}=0 \text {, }
$$

$$
\frac{d\left(r_{0} Q_{0}\right)}{d s_{0}}-r_{0} N_{s o}\left(\frac{1}{R_{0}}+\kappa_{s o}\right)-N_{\theta_{0}} \sin \phi+r_{0} \bar{p}_{0}=0 \text {, and }
$$

$$
\frac{d\left(r_{0} M_{s 0}\right)}{d s_{0}}+\left(r_{0} N_{s o} \sin \gamma-r_{0} Q_{0} \cos \gamma\right)\left(1+\varepsilon_{s o}\right)-M_{\theta 0} \cos t+P_{0} \sin \phi=0,
$$

where

$$
\bar{S}_{0}=S_{0} \cos \gamma-p_{0} \sin \gamma, \text { and }
$$

6 


$$
\bar{p}_{0}=S_{0} \sin \gamma+p_{0} \cos \gamma
$$

When the force equilibrium equations are rotated from meridional and normal to horizontal and vertical, the eisner equilibrium equations are obtained as

$$
\frac{d\left(r_{0} H_{0}\right)}{d s_{0}}-N_{\theta_{0}}+r_{0} p_{H_{0}}=0 \text {, and } \frac{d\left(r_{0} V_{0}\right)}{d s_{0}}+r_{0} p_{V_{0}}=0 \text {, }
$$

where

$$
\begin{aligned}
& P_{H_{0}}=S_{0} \cos (\phi-\gamma)+p_{0} \sin (\phi-\gamma), p_{V_{0}}=-S_{0} \sin (\phi-\gamma)+p_{0} \cos (\phi-\gamma), \\
& H_{0}=Q_{0} \sin \phi+N_{S O} \cos \phi \text {, and } V_{0}=Q_{0} \cos \phi-N_{s o} \sin \phi .
\end{aligned}
$$

The virtual work as presented by Reissner in Refs. 1 and 2 is

$$
\int_{L_{0}}\left\{N_{s o}\right\}^{\top} \delta\left\{\hat{E}_{s o}\right\} . r_{0} d s_{0}=\int_{L_{0}}\left\{p_{H_{0}}\right\}^{\top} \delta\left\{u_{r}\right\} r_{0} d s_{0}+\left[\left\{\bar{H}_{0}\right\}^{\top} \delta\left\{u_{r}\right\} r_{0}\right]_{B_{0}},
$$

where

$$
\begin{aligned}
& \left\{N_{s o}\right\}^{\top}=\left(N_{s o} N_{\theta 0} Q_{0} M_{s o} M_{\theta 0} P_{0}\right) \text { stress resultants and bending moments, } \\
& \left\{\hat{\varepsilon}_{\text {so }}\right\}^{\top}=\left(\begin{array}{llllll}
\hat{\varepsilon}_{\text {so }} & \varepsilon_{\theta_{0}} & \hat{\gamma}_{0} & \kappa_{\text {so }} & \kappa_{\theta_{0}} & \lambda_{0}
\end{array}\right) \text { membrane and bending strains, } \\
& \left\{p_{H o}\right\}^{\top}=\left(p_{H o} p_{\text {vo }} 0\right) \text { initial surface loads, } \\
& \left\{u_{r}\right\}^{T}=\left(u_{r} u_{z} \beta\right) \text { displacements and meridional rotation, and } \\
& \left\{\left.\bar{H}_{0}\right|^{\top}=\left(\bar{H}_{0} \bar{V}_{0} \bar{M}_{s o}\right)\right. \text { applied stress resultants and applied bending moment. }
\end{aligned}
$$

7 
- $\bar{H}_{0}$ and $\bar{V}_{0}$ are applied stress resultants, and $\bar{M}_{\text {so }}$ is an applied bending moment.

- $L_{0}$ is the undeformed length of the neutral surface, and $B_{0}$ represents both boundaries at the ends of the shell.

- $u_{r}$ and $u_{z}$ are the vertical (radial) and horizontal (axial) displacements.

- $B$ is the rotation $\phi-\phi_{0}$.

- $\delta$ is the variational operator.

- The variations of the strains are

$$
\begin{aligned}
& \delta \hat{\varepsilon}_{s o}=\cos \phi \frac{d\left(\delta u_{r}\right)}{d s_{0}}-\sin \phi \frac{d\left(\delta u_{2}\right)}{d s_{0}}-\frac{q}{q_{0}} \sin \gamma \delta \beta, \quad \delta \varepsilon_{\theta_{0}}=\frac{\delta u_{r}}{r_{0}}, \\
& \delta \hat{r}_{0}=\sin \phi \frac{d\left(\delta u_{r}\right)}{d s_{0}}+\cos \phi \frac{d\left(\delta u_{z}\right)}{d s_{0}}+\frac{q}{q_{0}} \cos \gamma \delta \beta, \quad \delta \kappa_{s o}=\frac{d(\delta \beta)}{d s_{0}}, \\
& \delta \kappa_{\theta_{0}}=\frac{\cos \phi}{r_{0}} \delta \beta, \quad \text { and } \delta \lambda_{0}=-\frac{\sin \phi}{r_{0}} \delta \beta .
\end{aligned}
$$

- The strains are

$$
\hat{\varepsilon}_{s 0}=\cos \phi \frac{d u_{r}}{d s_{0}}-\sin \phi \frac{d u_{z}}{d s_{0}}+\cos \beta-1=\left(1+\varepsilon_{s 0}\right) \cos \gamma-1,
$$

where $\varepsilon_{\text {so }}=\frac{q-q_{0}}{q_{0}}, \quad \varepsilon_{\theta_{0}}=\frac{r-r_{0}}{r_{0}}=\frac{u_{r}}{r_{0}}$,

$$
\begin{aligned}
& \hat{p}_{0}=\sin \phi \frac{d u_{r}}{d s_{0}}+\cos \phi \frac{d u}{d s_{0}}+\sin \beta=\left(1+\varepsilon_{s 0}\right) \sin \gamma, \quad \kappa_{s o}=\frac{d \beta}{d s_{0}}, \\
& \kappa_{\theta_{0}}=\frac{\sin \phi-\sin \phi_{0}}{r_{0}}, \text { and } \lambda_{0}=\frac{\cos \phi-\cos \phi_{0}}{r_{0}} .
\end{aligned}
$$




\section{IIi. NONLINEAR SHELL-OF-REVOLUTION MODEL}

The computational technique used to develop this nonlinear shell-ofrevolution model consists of the following four steps.

- Modify the virtual work that satisfies equilibrium for incremental loadings.

- Lineziize the virtual work so that it may be solved directly.

- Use the finite element method to approximate the linearized incremental virtual work. This step is identical to solving a linear problem.

- By iteration, approximate the original nonlinear virtual work. This iteration is necessary because the virtual work was linearized, and iteration ensures that equilibrium is satisfied. The iteration step is identical to the increment step except that the applied loads do not change. Also, this iteration step makes it possible to include nonlinear materials.

The incremental loads for the $j$ increment are defined as

$\left\{\Delta \mathbf{P}_{H_{0}}\right\}_{j}=\left\{\mathbf{P}_{H_{0}}\right\}_{j+1}-\left\{\mathbf{P}_{H_{0}}\right\}_{j} \quad$ and $\quad\left\{\Delta \bar{H}_{0}\right\}_{j}=\left\{\bar{H}_{0}\right\}_{j+1}-\left\{\bar{H}_{0}\right\}_{j}$.

The incremental displacements, the incremental stress resultants and bending moments, and the incremental membrane and bending strains for the $j$ increment are def ined as

$$
\begin{aligned}
& \left\{\Delta u_{r}\right\}_{j}=\left\{u_{r}\right\}_{j+1}-\left\{u_{r}\right\}_{j}, \quad\left\{\Delta N_{s o}\right\}_{j}=\left\{N_{s o}\right\}_{j+1}-\left\{N_{s o}\right\}_{j}, \text { and } \\
& \left\{\hat{s}_{s o}\right\}_{j}=\left\{\hat{\varepsilon}_{s o}\right\}_{j+1}-\left\{\hat{\varepsilon}_{s o}\right\}_{j} .
\end{aligned}
$$

Thus the virtual work for the $j$ increment can be written as

$$
\left.\left.\int_{L_{0}}\left\{\Delta N_{s o}\right\}_{j}^{\top} \delta\left\{\Delta \hat{\varepsilon}_{s o}\right\} j r_{0} d s_{0}=-\int_{L_{0}} \int_{N_{s o}}\right\}_{j}^{T} \delta\right\}_{\left.\Delta \hat{\varepsilon}_{s o}\right\} j} r_{0} d s_{0}
$$




$$
\int_{L_{0}}\left\{p_{H_{0}}{ }_{j+1}^{T} \delta\left\{\Delta u_{r}\right\}_{j} r_{0} d s_{0}+\left[r_{0}\left\{\bar{H}_{0}\right\}_{j+1} \delta\left\{\Delta u_{r}\right\}_{j}\right] B_{0} .\right.
$$

This equation can be linearized by assuming $\left(\Delta u_{r}\right)_{j},\left(\Delta u_{z}\right)_{j}$, and $(\Delta \beta)_{j}$ are sma 11. Thus the incremental strains are $\left(\left(\phi_{j},\left(\hat{P}_{0}\right)_{j}\right.\right.$, and $\left(\hat{\varepsilon}_{\text {so }}\right)_{j}$ are known from the last increment)

$$
\begin{aligned}
& \left(\Delta \hat{\varepsilon}_{s_{0}}\right)_{j}=\cos (\phi)_{j} \frac{d\left(\Delta u_{r}\right)_{j}}{d s_{0}}-\sin (\phi)_{j} \frac{d\left(\Delta u_{z}\right)_{j}}{d s_{0}}-\left(\vartheta_{0}\right)_{j}(\Delta B)_{j},\left(\Delta \varepsilon_{\theta 0}\right)_{j}=\frac{\left(\Delta u_{r}\right)_{j}}{r_{0}}, \\
& \left(\Delta \hat{q}_{0}\right)_{j}=\sin (\phi)_{j} \frac{d\left(\Delta u_{r}\right)_{j}}{d s_{0}}+\cos (\phi)_{j} \frac{d\left(\Delta u_{z}\right)_{j}}{d s_{0}}+\left(\left(\hat{\varepsilon}_{s o}\right)_{j}+1\right)(\Delta \beta)_{j},
\end{aligned}
$$

$\left(\Delta \kappa_{s o}\right)_{i}=\frac{d(\Delta \beta)_{j}}{d s_{0}}, \quad\left(\Delta \kappa_{\theta_{0}}\right)_{j}=\frac{\cos (\phi)_{j}}{r_{0}}(\Delta \beta)_{j}, \quad$ and $\quad\left(\Delta \lambda_{0}\right)_{j}=-\frac{\sin (\phi)_{j}}{r_{0}}(\Delta \beta)_{j}$. As sume the following constitutive relations:

$$
\left\{N_{s o}\right\}_{j}=[D]_{s j}\left\{\hat{\varepsilon}_{s o}\right\}_{j}+\left[N_{s o i}\right\}_{j} \quad \text { and } \quad\left\{\Delta N_{s o}\right\}_{j}=[D]_{t j}\left\{\Delta \hat{\varepsilon}_{s o}\right\}_{j}+\left\{\Delta N_{s o j}\right\}_{j},
$$

where

$[D]_{s j}$ and $[D]_{t j}$ are the secant and tangent material matrix for the $j$ increment. An orthotropic material would have the following form for the material matrix.

$$
[D]=\left[\begin{array}{llllll}
c_{s} & C_{s e} & 0 & T_{s} & T_{s \theta} & 0 \\
C_{s \theta} & C_{\theta} & 0 & T_{s \theta} & T_{\theta} & 0 \\
0 & 0 & G & 0 & 0 & 0 \\
T_{S} & T_{s \theta} & 0 & D_{s} & D_{s \theta} & 0 \\
T_{s} \theta & T_{\theta} & 0 & D_{s \theta} & D_{\theta} & 0 \\
0 & 0 & 0 & 0 & 0 & D_{\lambda}
\end{array}\right]
$$


Lack ing a good feel for $D_{\lambda}$, I decided to use $D_{\lambda}=D_{\theta}$, because $P_{0}$ is a bending moment that uses hoop stress as does $\kappa_{\theta 0}$. The other terms of the material matrix are discussed in Appendixes $B, C$, and $D .\left\{\mathbb{N}_{\text {so } i}\right\}_{j}$ and $\left\{\mathbb{W}_{\text {soi }}\right\}_{j}$ are the initial stress resultants and initial bending moments (thermal stresses can be included through these matrices).

These are defined as

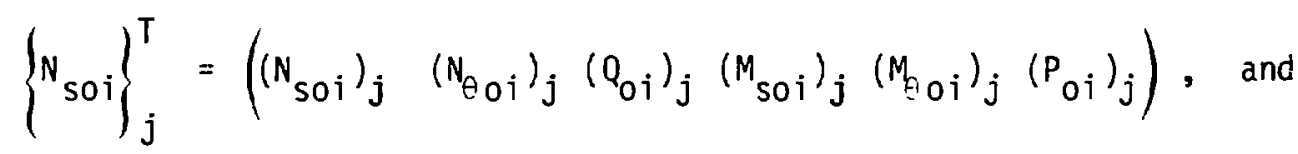

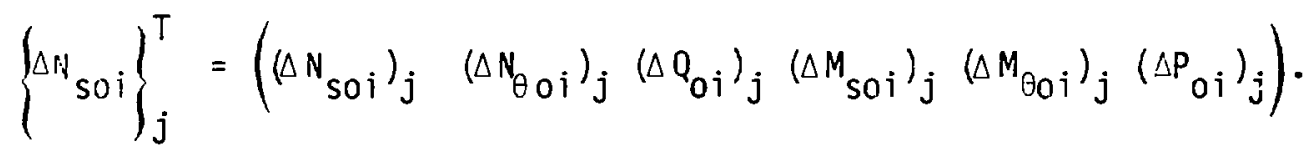

Thus the incremental virtual work for the $j$ increment can be written as

$$
\begin{aligned}
& \int_{L_{0}}\left\{\hat{\varepsilon}_{s o}\right\}_{j}^{\top}[0]_{t j} \delta\left\{\Delta \hat{\varepsilon} s_{s o}\right\} j r_{0} d s_{0}=-\int_{L_{0}}\left(\left\{N_{s o}\right\}^{\top}+\left\{\Delta N_{s o i}\right\}_{j}^{T}\right) \delta\left\{\Delta \hat{\varepsilon}_{s o}\right\} j r_{0} d s_{0} \\
& +\int_{L_{0}}\left\{p_{H o}\right\}_{j+1}^{T} \delta\left\{u_{r}\right\} j r_{0} d s_{0}+\left[r_{0}\left\{\bar{H}_{0}\right\}_{j+1}^{T} \delta\left\{u_{r}\right\} j\right] B_{0} .
\end{aligned}
$$

Again, the iteration step is the same as the incremental step except the loads $\left\{p_{H_{0}}\right\}_{j+1}$ and $\left\{\bar{H}_{0}\right\}_{j+1}$ do not change. Also, the material matrix $[0]_{t j}$ can change for each iteration.

\section{FINITE ELEMENT APPROXIMATION}

The finite element approximation for this shell of revolution can be either two- or three-nodal point elements.

Thus the approximations for displacements and the rotation are 
$\Delta u_{r}=\sum_{i=1}^{m} h_{m i} a_{r i}, \quad \Delta u_{z}=\sum_{i=1}^{m} h_{m i} a_{z i}, \quad$ and $\Delta \beta=\sum_{i=1}^{m} h_{m i} a_{B i}$,

where $m$ is the rumber of nodal points in this element and $h_{m i}$ are the shape functions. For two nodal point elements,

$h_{21}=\frac{1}{2}(1+\xi)$ and $h_{22}=\frac{1}{2}(1-\xi)$.

For three nodal point elements,

$h_{31}=\frac{1}{2} \xi(1+\xi), \quad h_{32}=-\frac{1}{2} \xi(1-\xi), \quad$ and $\quad h_{33}=1-\xi^{2}$.

For isoparametric elemeris, $r_{0}$ and $z_{0}$ are approximated with the same shape functions.

$r_{0}=\sum_{i=1}^{m} h_{m i} r_{0 i}=r_{0}(\xi)$, and $z_{0}=\sum_{i=1}^{m} h_{m i} z_{0 i}=z_{0}(\xi)$;

then

$q_{0}=q_{0}(\xi)=\left[\left(\frac{d r_{0}}{d \xi}\right)^{2}+\left(\frac{d z_{0}}{d \xi}\right)^{2}\right]^{1 / 2}=\left[\left(\sum_{i=1}^{m} \frac{d h_{m i}}{d \xi} r_{0 i}\right)^{2}+\left(\sum_{i=1}^{m} \frac{d h_{m i}}{d \xi} z_{0 i}\right)^{2}\right]^{1 / 2}$.

Thus $r_{0} d s_{0}$ is $r_{0}(\xi) q_{0}(\xi) d \xi$ where $-1 \leq \xi \leq 1$. In matrix notation

$\left\{\Delta u_{r}\right\}_{j}^{T}=[H(\xi)]\left\{a_{r 1}\right\}_{j}$,

where

$\left\{\Delta a_{r 1}\right\}_{j}^{\top}=\left(\begin{array}{llll}\left(a_{r 1}\right)_{j} & \left(a_{z 1}\right)_{j} & \left(a_{B 1}\right)_{j} \ldots & \left(a_{B m}\right)\end{array}\right) \quad$ and 


$H(\xi)=\left[\begin{array}{lllllll}h_{m 1} & \cdot & \cdot & \cdot & h_{m m} & 0 & 0 \\ 0 & \cdot & \cdot & \cdot & 0 & h_{m m} & 0 \\ 0 & \cdot & \cdot & \cdot & 0 & 0 & h_{m m}\end{array}\right]$.

Also

$\left\{\Delta \hat{\varepsilon}_{s o}\right\}_{j}^{\top}=[B]_{j} \quad\left\{a_{r l}\right\}_{j}$,

where $[B]_{j}$ is obtained us ing the linearized form of $\left\{\Delta \hat{\varepsilon}_{s o}\right\}_{j}$ and the finite element approximations for $\left\{\Delta_{r}\right\}_{j}$.

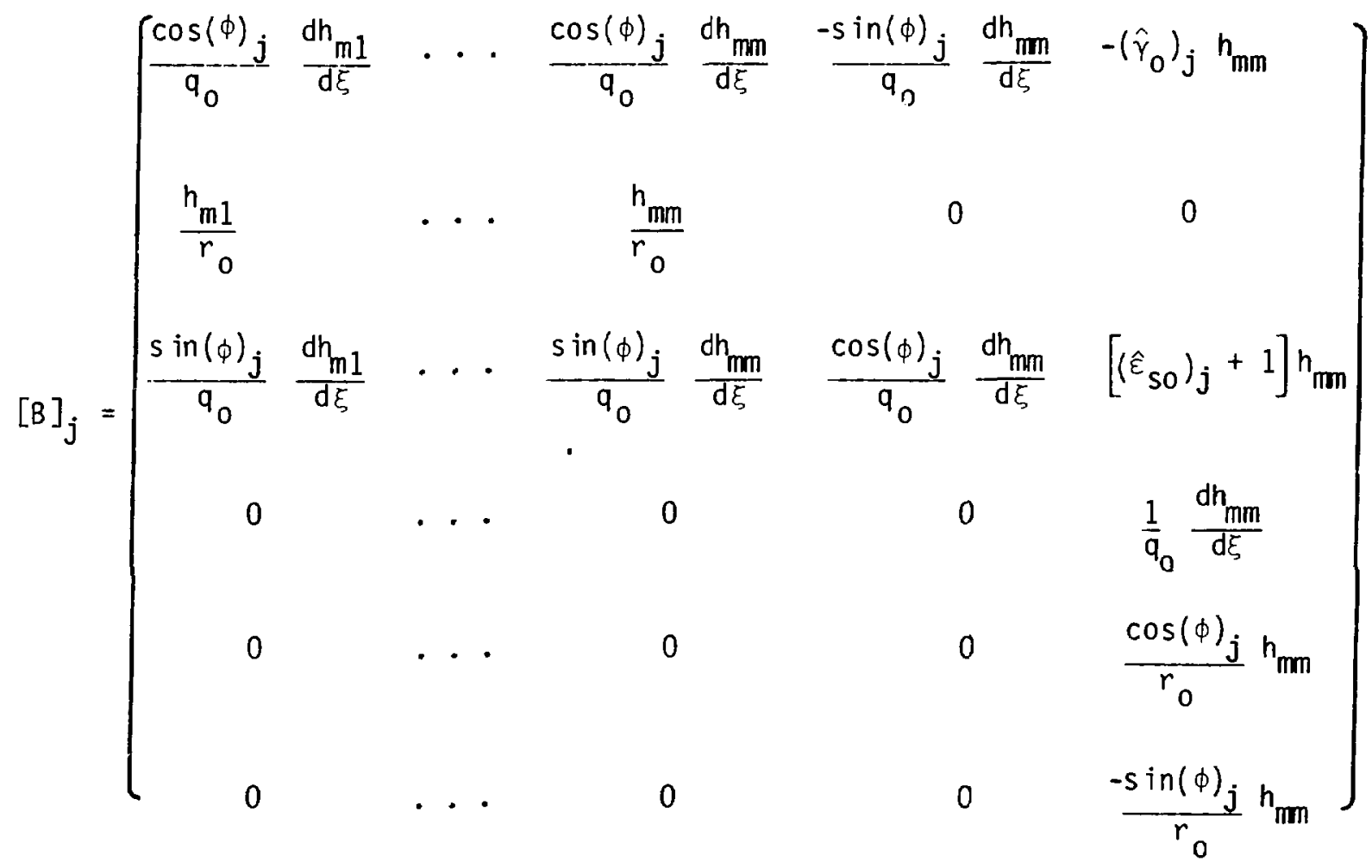

With the finite element approximations presented, the incremental virtual work may be written

$\sum_{k=1}^{K}\left\{a_{r 1}\right\}_{j k}^{T}[k]_{j k}\left\{a_{r l}\right\}_{j k}-\{F\}_{j k}^{T}\left\{a_{r 1}\right\}_{j k}=0$ 
where

$K$ is the number of elements used in the problem;

$\left\{a_{r l}\right\}_{j k}^{\top}$ is $\left\{a_{r 1}\right\}_{j}^{\top}$ for the k element

$[k]_{j k}=\left(\int_{-1}^{1}[B]_{j}^{\top}[D]_{t j}[B]_{j} r_{0}(\xi) q_{0}(\xi) d \xi\right)_{k}$

and is the stiffness matrix for the $k$ element; and

$$
\begin{aligned}
\{F\}_{j k}^{T} & =\left(\int_{-1}^{1}\left\{p_{H_{0}}\right\}_{j+1}^{\top}[H(\xi)] r_{0}(\xi) q_{0}(\xi) d \xi\right. \\
& -\int_{-1}^{1}\left(\left\{{ }^{N}{ }_{s o}\right\}_{j}^{T}+\left\{\Delta N_{s o i}\right\}_{j}^{T}\right)[B]_{j} r_{0}(\xi) q_{0}(\xi) d \xi \\
& + \text { (Boundary applied forces and moments) })_{k}
\end{aligned}
$$

and is the force vector for the $k$ element.

\section{VER IFICATION PROBLEMS}

In this section I describe the problems I used to check this model.

Grafton and Strome developed a finite element shell-of-revolution code in the early sixties. In their paper (Ref. 5) they checked their linear code with several example problems. I used the nonlinear model described in this report to solve three of these.

(1) A circular plate with a shear load on the boundary of an interior hole and the outer boundary fixed. This problem was modeled with 21 equally spaced nodal points and is illustrated in Fig. 4. 


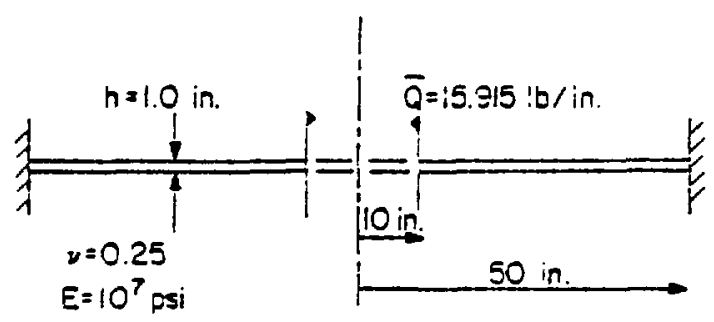

Fig. 4.

Circular plate with shear loading.
(2) A cylindrical shell with a shear load on one end and the other end fixed. This problem used 25 nodal points with a very fine spacing next to the shear load and the space between nodal points increasing to a coarse spacing next to the fixed boundary edge. This problem is illustrated in Fig. 5 .

(3) A $60^{\circ}$ segment of a hemisphere. At one boundary edge the radial coordinate was one half of the radius of the hemisphere. This boundary edge was loaded with an applied moment, and the other boundary was fixed. Again, the spacing of nodal points was very fine next to the applied moment and increasing to coarse next to the fixed boundary. There were 29 nodal points as illustrated in Fig. 6.

This model solved all three linear problems in a nonlinear mode with exact integration and converged to the linear solutions using both two- and threenodal-point elements. It also solved all three linear problems in a linear mode (one increment and no iterations). The three-nodal-point elements were accurate with both exact or reduced integration, whereas the two-nodal-point elements were only accurate with reduced integration or exact integration with many more nodal points than those illustrated in Figs. 4, 5, and 6 . Reference 6 compares the solution of these three problems using this model with other models.

The next two example problems were solved to check whether this model could effectively calculate large membrane strains. See Appendix $E$ for theoretical calculations for these two problems.

(1) An axialiy loaded cylinder, which is shown in Fig. 7. This problem, which degenerates to a linear solution, can be solved with one increment and does not require any equilibrium iteration. Only four nodal points were used for this problem, and the ariswers were exact.

(2) A pressure-loaded hemisphere, which is shown in Fig. 8. This problem was modeled with 9 nodal points and solved with one load increment and one equilibrium iteration. The maximum error in the displacements was $0.42 \%$. Additional iterations changed the answers only slightly. 


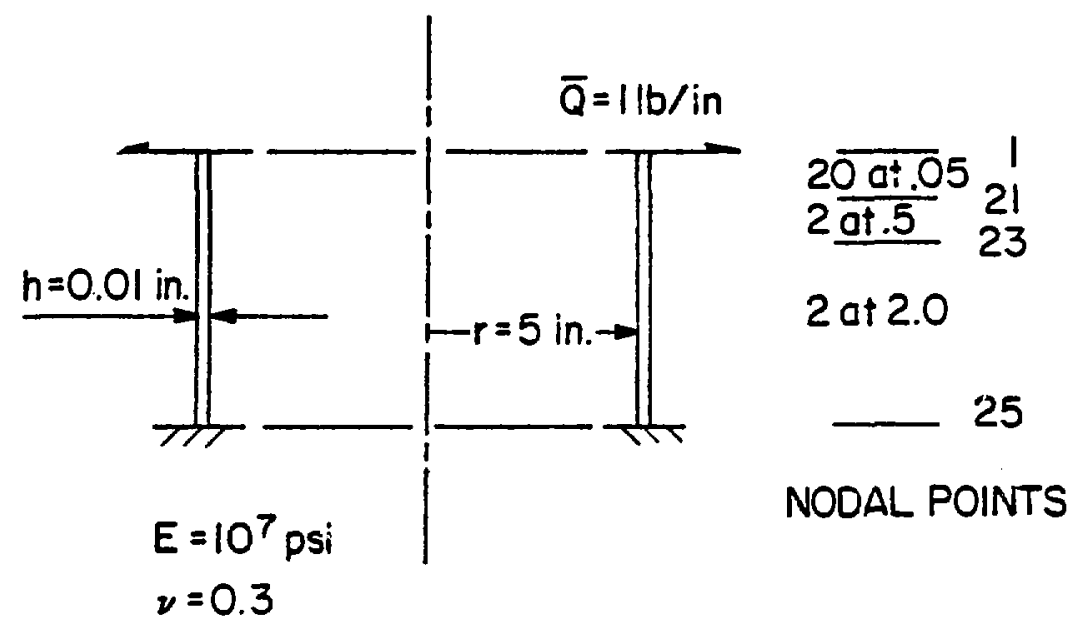

Fig. 5 .

Cylindrical shell with shear loading.

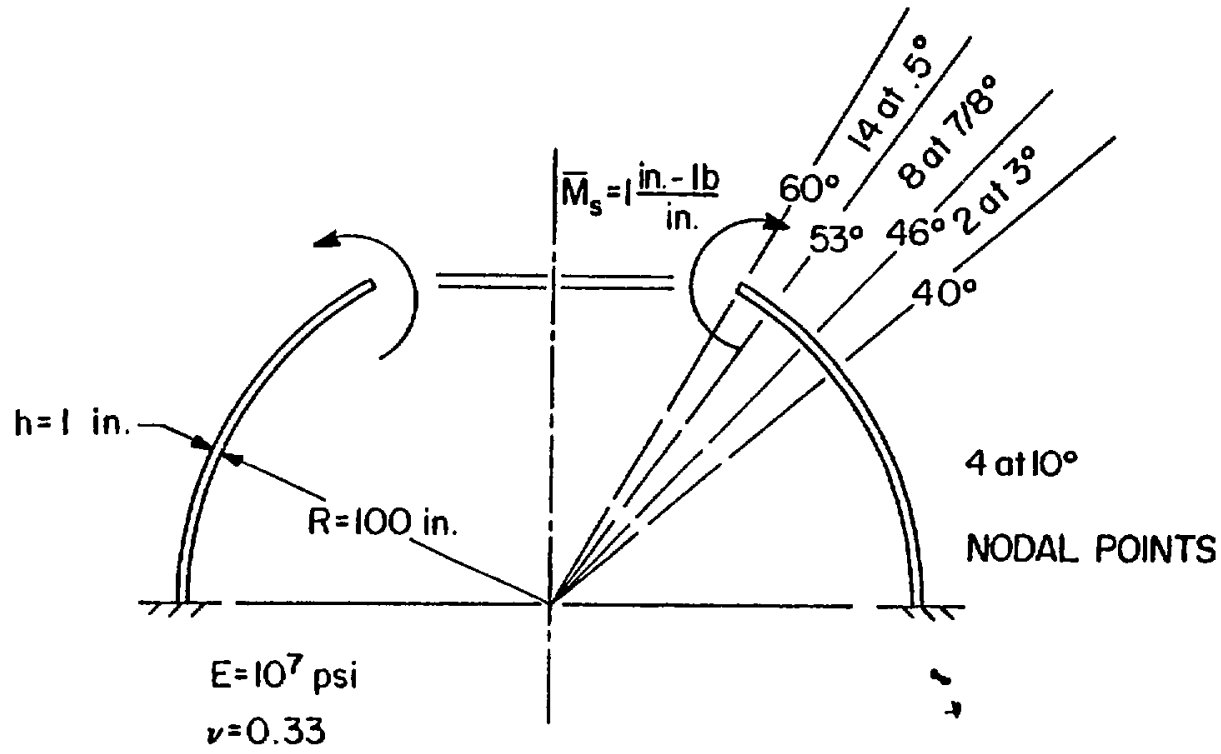

Fig. 6.

Hemisphericil shell with an applied bending moment. 


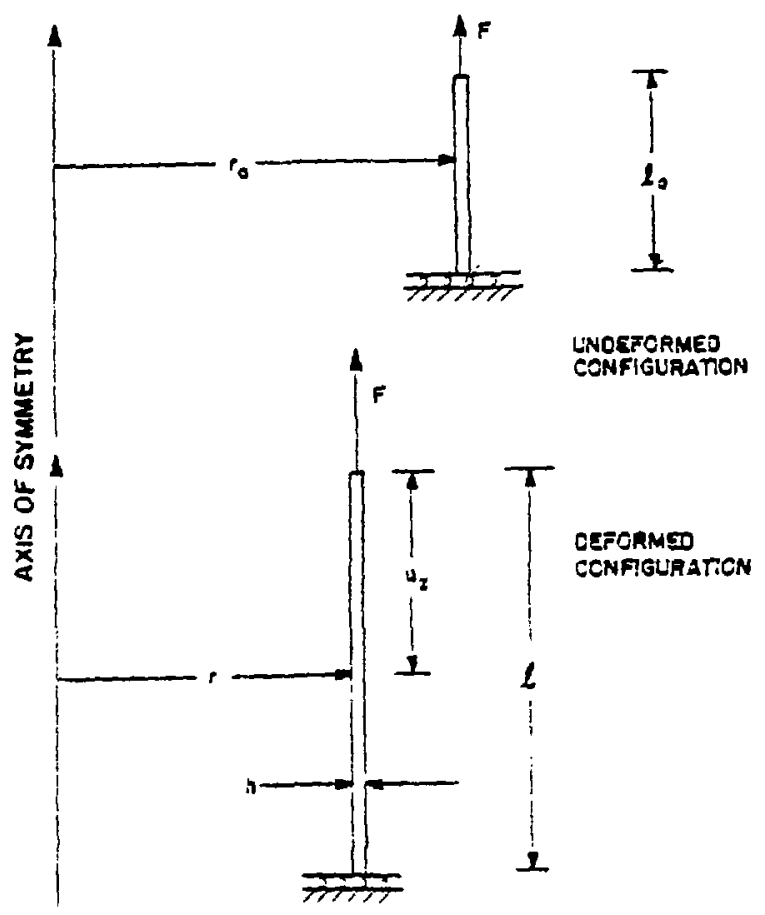

Fig. 7.

Axially loaded cylindrical problem.

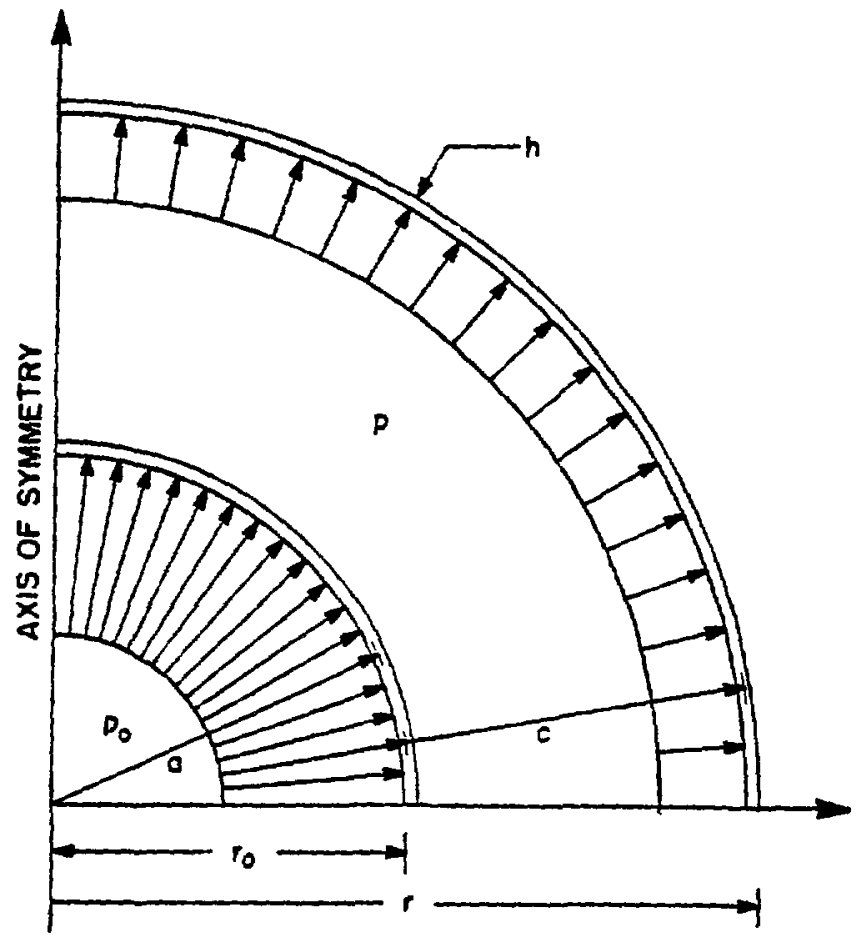

Fig. 8.

Hemisphere with thermal and pressure loadings. 
These problems were solved with both two- and three-nodal-point elements and demonstrate the ability of this model to calculate large membrane strains.

The last problem solved was to load a cylinder such that it deforms into a portion of a hemisphere. Appendix $E$ describes the load calculations that were required for this protlem. This problem is shown in Fig. 9 and demonstrates the ability of this model to calculate large rotations. This problem was modeled with 21 nodal points and was solved with one load increment. See Fig. 10 for a graphical illustration of the error in the rotation $\beta$ vs number of iterations. The error in the rotation was much higher than the errors in the displacements. When I used a shear modulus $G$ calculated from $\frac{E}{2(1+\nu)}$ for th is problem, the solution diverged in the iteration portion of the solution schene for both two- and three-nodal-point elements. By increasing the shear modulus to $G=40 E$, the problem converged for two-nodal-point elements. I also found that

(1) if $\gamma^{\prime}$ 's are set to zero, the solution scheme diverges during iteration;

(2) if $\gamma^{\prime} s$ are set to zero after the first four iterations, the iteration scheme converges to the correct answers; and

(3) If $\gamma^{\prime}$ s are set to their theoretical values, the solution scheme again diverges during iteration.

Thus, for this problem, $\gamma^{\prime} s$ are very small and have no effect on the final answers but are necessary for the solution scheme to converge during iteration. This is typical; as the thickness decreases, shear deformations have less effect, but the conditioning for iteration is poorer. Improved iteration schemes (Ref. 7) do exist, and I expect that these would enhance the perforinance of this mode 1 .

VI. CONCLUSIONS AND RECOMMENDATIONS

The finite element nonlinear shell model described in this report was developed to solve

- linear problems,

- problems with large membrane strains,

- problems with large rotations, and

- problems with nonlinear materials.

As shown in Sec. $V$, this model accurately solves linear problems, large membrane problems, and large rotations problems. However, the iteration scheme 

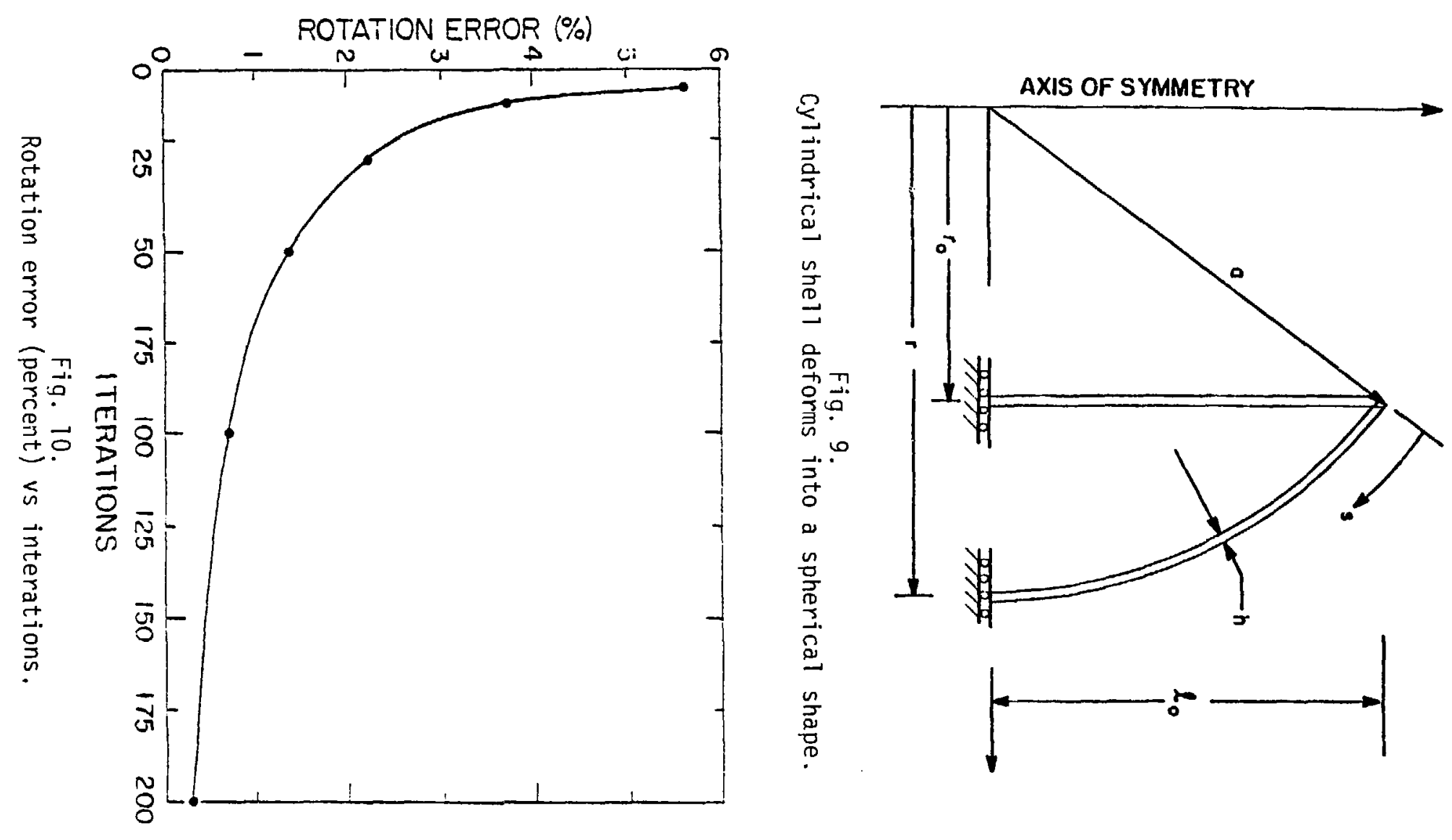
does not always converge for large rotations. In Ref. 8 th is model was used for a nonlinear material problem and severe dynamic loads, and again stajility problems halted the solution.

Thus I recommend that improved iteration schemes be investigated for this model such as those described in Ref. 7. Until that is done: I suggest that a large shear modulus be used to improve the stability of the solution scheme.

Also, remember that these nonlinear large rotations problems $\left(50^{\circ}\right.$ rotation) are very difficult problems, and many researciers are searching for general techniques to solve these nonlinear problems.

One of the basic as sumptions used in shell theory is to assume zero normal stresses. This assumption is valid when the normal pressure is small compared to the material moduli. This as sumption was checked for the example problems illustrated in Figs. 8 and 9 (see Appendix E). In both cases, the error was less than $5 \%$. Eliminating this as sumption is very similar to adding the thermal strain effect and could easily be done on all problems except those where continuum elements are effecting the normal pressure on the shell. Then the programming would be quite involved.

APPENDIX A EQUILIBR IUM EQUATIONS

There are three equilibrium equations for a symetrically loaded shell of revolution with shear deformation. These are equilibrium along the meridional coordinate $s$, the nurmal coordinate $n$, and rotational equilibrium.

Fram Figs. 2--3 and $A-1--A-3$, the meridional equilibrium equation is

$$
\begin{aligned}
& \left(N_{S}+\Delta N_{S}\right)(r+\Delta r) \Delta \theta \cos \left(\gamma+\frac{\Delta \gamma}{2}+\frac{\Delta \phi}{2}\right)-N_{S} r \Delta \theta \cos \left(\gamma+\frac{\Delta \gamma}{2}-\frac{\Delta \phi}{2}\right) \\
& +(Q+\Delta Q)(r+\Delta r) \Delta \theta \sin \left(\gamma+\frac{\Delta \gamma}{2}+\frac{\Delta \phi}{2}\right)-Q r \Delta \theta \sin \left(\gamma+\frac{\Delta \gamma}{2}-\frac{\Delta \phi}{2}\right) \\
& -2 N_{\theta} \Delta s \sin \left(\frac{\Delta \theta}{2}\right) \cos \left(\phi-\gamma+\frac{\Delta \phi}{2}-\frac{\Delta \gamma}{2}\right)+S\left(r+\frac{\Delta r}{2}\right) \Delta \theta \Delta s=0 .
\end{aligned}
$$




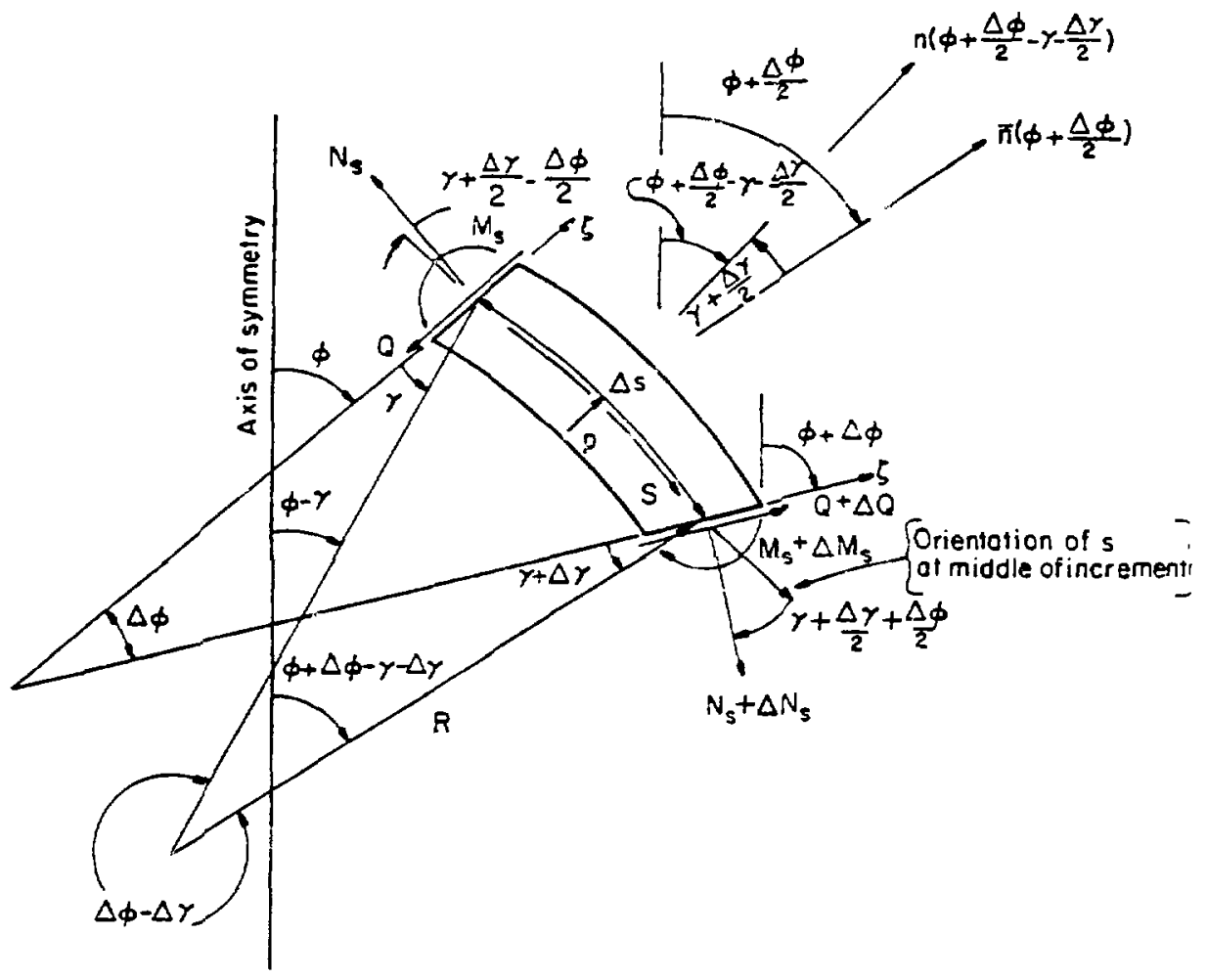

Fig. A-1.

Meridional cross-sectional view of incremental element ( $\theta$ is constant in Fig. 2).

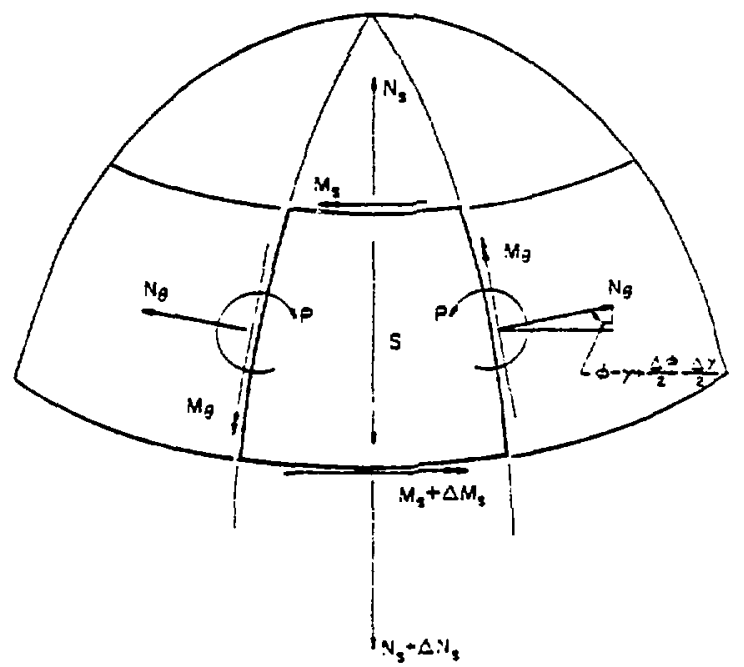

Fig. A-2.

Normal view of incremental element.

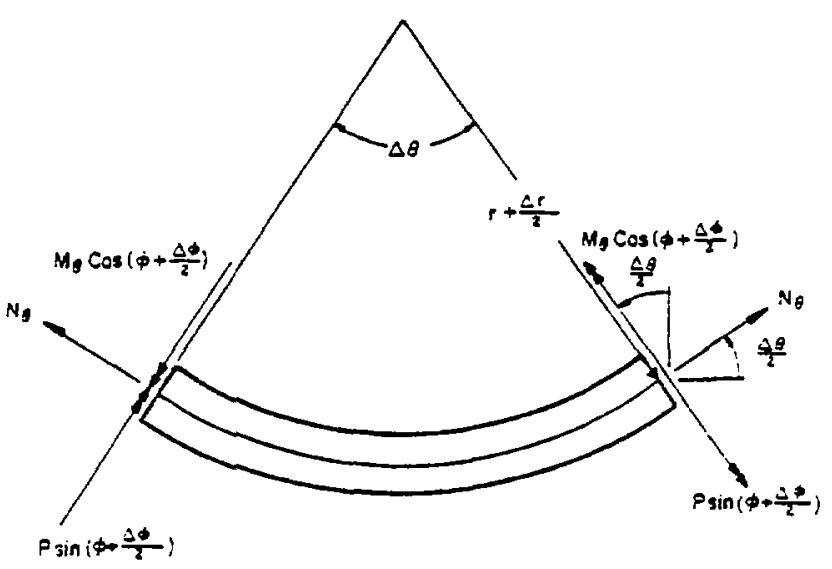

Fig. A-3.

Circumferential cross-sectional view of incremental element ( $\phi$ is a constant in Fig. 2). 
As $\Delta \phi-\Delta Y$ becomes small and $\Delta a$ becomes small,

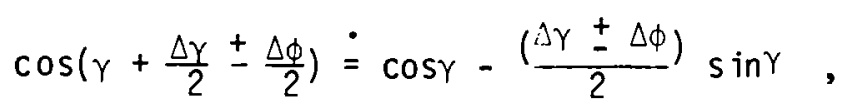

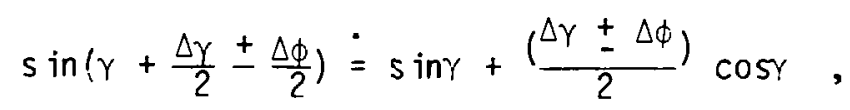

$\cos \left(\zeta-\gamma+\frac{\Delta \phi}{2}-\frac{\Delta \gamma}{2}\right) \dot{=} \cos (\phi-\gamma)-\frac{(\Delta \phi-\Delta \gamma)}{2} \sin (\phi-\gamma)$, and $\sin \left(\frac{\Delta \theta}{2}\right)=\frac{\Delta \theta}{2}$

Substitute Eq. (A-2) into Eq. (A-1) and ignore terms with more than two incremental quantities, as these will go to zero in the limit.

$-N_{S} r \sin \gamma \Delta \phi \Delta \theta+\left(N_{S} \Delta r+r \Delta N_{S}\right) \Delta \theta \cos \gamma+Q r \cos \gamma \Delta \phi \Delta \theta$

$+(Q \Delta r+r \Delta Q) \Delta \theta \sin \gamma-N_{\theta} \cos (\phi-\gamma) \Delta \theta \Delta s+S r \Delta \theta \Delta s=0$

Dividing this equation by $\Delta s \Delta \theta$ :

$\frac{\left(N_{S} \Delta r+r \Delta N_{S}\right)}{\Delta S} \cos \gamma-N_{S} r \frac{\Delta \phi}{\Delta S} \sin \gamma+\frac{(Q \Delta r+r \Delta Q)}{\Delta S} \sin \gamma+Q r \frac{\Delta \phi}{\Delta S} \cos \gamma$

$-N_{\theta} \cos (\phi-\gamma)+r S=0$.

In the limit, this equation can be written as

$\frac{d\left(r N_{s}\right)}{d s} \cos \gamma-N_{s} r \frac{d \phi}{d s} \sin \gamma+\frac{d(r Q)}{d s} \sin \gamma+Q r \frac{d \phi}{d s} \cos \gamma-N_{\theta} \cos (\phi-\gamma)+r s=0$.

Also, from Figs. $2--3$ and $A-1--A-3$, the normal equilibrium equation is 


$$
\begin{aligned}
& N_{S} r \Delta \theta \sin \left(\gamma+\frac{\Delta \gamma}{2}-\frac{\Delta \phi}{2}\right)-\left(N_{S}+\Delta N_{S}\right)(r+\Delta r) \Delta \theta \sin \left(\gamma+\frac{\Delta y}{2}+\frac{\Delta \phi}{2}\right) \\
& +(Q+\Delta Q)(r+i r) \Delta \theta \cos \left(\gamma+\frac{\Delta \gamma}{2}+\frac{\Delta \phi}{2}\right)-Q r \Delta \theta \cos \left(\gamma+\frac{\Delta y}{2}-\frac{\Delta \phi}{2}\right) \\
& -2 N_{0} \Delta s \sin \left(\frac{\Delta \theta}{2}\right) \sin \left(\phi-\gamma+\frac{\Delta \phi}{2}-\frac{\Delta \gamma}{2}\right)+p\left(r+\frac{\Delta r}{2}\right) \Delta \theta \Delta s=0 .
\end{aligned}
$$

In a manner similar to the meridional equilibrium equation, this normal equilibrim equation can be written as

$$
\begin{aligned}
& -\frac{d\left(r N_{s}\right)}{d s} \sin \gamma-N_{s} r \frac{d \phi}{d s} \cos \gamma+\frac{d(r Q)}{d s} \cos \gamma-Q r \frac{d \phi}{d s} \sin \gamma-N_{\theta} \sin (\phi-\gamma) \\
& \quad+r p=0 .
\end{aligned}
$$

The meridional equilibrium Eq. (A-5) and the normal equilibrium Eq. (A-7) can be rotated by the angle $\gamma$ to give

$$
\frac{d\left(r N_{s}\right)}{d s}+r Q \frac{d \phi}{d s}-N_{\theta} \cos \phi+r \bar{S}=0 \text { and } \frac{d(r Q)}{d s}-r N_{s} \frac{d \phi}{d s}-N_{\theta} \sin \phi+r \bar{p}=0
$$

where $\bar{S}=S \cos \gamma-p \sin \gamma$ and $\bar{p}=S \sin \gamma+p \cos \gamma$.

The rotational equilibrium equation is derived using Figs. 2--3 and $A-1--A-4$ as

$$
\begin{aligned}
& \left(N_{S}+\Delta N_{S}\right)(r+\Delta r) \Delta \theta \sin \left(\gamma+\frac{\Delta \gamma}{2}+\frac{\Delta \phi}{2}\right) R \sin \left(\frac{\Delta \phi}{2}-\frac{\Delta \gamma}{2}\right) \\
& +N_{S} r \Delta \theta \sin \left(\gamma+\frac{\Delta \gamma}{2}-\frac{\Delta \phi}{2}\right) R \sin \left(\frac{\Delta \phi}{2}-\frac{\Delta \gamma}{2}\right) \\
& -(Q+\Delta Q)(r+\Delta r) \Delta \theta \cos \left(\gamma+\frac{\Delta \gamma}{2}+\frac{\Delta \phi}{2}\right) R \sin \left(\frac{\Delta \phi}{2}-\frac{\Delta \gamma}{2}\right)
\end{aligned}
$$

23 


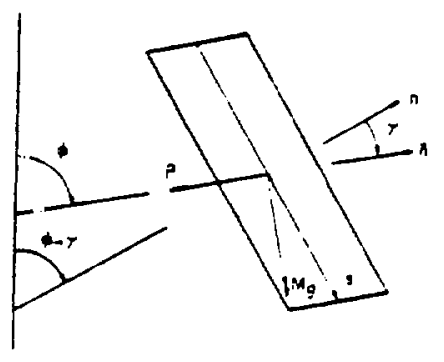

Fig, A-4.

Meridional sectional view of $M_{\theta}$ and $P$ for an incremental conical element $(\theta$ is constant).

$$
\begin{array}{rl}
-Q & r \Delta \theta \cos \left(\gamma+\frac{\Delta \gamma}{2}-\frac{\Delta \phi}{2}\right) R \sin \left(\frac{\Delta \phi}{2}-\frac{\Delta \gamma}{2}\right) \\
& +\left(M_{S}+\Delta M_{S}\right)(r+\Delta r) \Delta \theta
\end{array}
$$

$$
\begin{gathered}
-M_{s} r \Delta \theta-2 M_{\theta} \sin \left(\frac{\Delta \theta}{2}\right) \Delta s \cos \left(\phi+\frac{\Delta \phi}{2}\right) \\
+2 P \sin \left(\frac{\Delta \theta}{2}\right) \Delta s \sin \left(\phi+\frac{\Delta \phi}{2}\right)=0 .
\end{gathered}
$$

Again, using the approximation for $\Delta \phi-\Delta \gamma$ and $\Delta \theta$ becoming small angles (Eq. A-2), and because

$R \sin \left(\frac{\Delta \phi}{2}-\frac{\Delta \gamma}{2}\right) \dot{=} \Delta s$,

Eq. $(A-9)$ in the limit becomes

$$
\frac{d\left(r M_{s}\right)}{d s}+r N_{s} \sin \gamma-r Q \cos \gamma-M_{\theta} \cos \phi+P \sin \phi=0 .
$$

$(A-10)$

APPENDIX B

INTEGRATION THROUGH THE THICKNESS

In this appendix the rationale for integration through the thickness is explained. Consider the following constitutive equations.

$$
\begin{aligned}
& \sigma_{s o}=\bar{C}_{s} E_{s o}+\bar{C}_{s \theta} E_{\theta 0}, \\
& \sigma_{\theta 0}=\bar{C}_{s \theta} E_{s o}+\bar{C}_{\theta} E_{\theta 0}, \text { and } \\
& \tau_{0}=\bar{G} E_{s n o} .
\end{aligned}
$$

24 
$\sigma_{\text {so }}, \sigma_{\theta_{0}}$, and $\tau_{0}$ are Lagrangian, the meridional, hoop, and shearing stresses (Ref. 9). $E_{s o}, E_{\theta_{0}}$, and $E_{\text {sno }}$ are the meridional, hoop, and shearing strains. $\bar{C}_{S}, \bar{C}_{\theta}, \bar{C}_{s \theta}$, and $\bar{G}$ are orthotropic material properties.

The strains are (see Appendix $C$ )

$$
E_{\text {SO }}=\frac{\hat{\varepsilon}_{\text {SO }}+\zeta K_{\text {SO }}}{1+\zeta \frac{1}{R_{0}}} \text {, }
$$

$E_{\theta_{0}}=\frac{\varepsilon_{\theta_{0}}+\zeta_{\kappa} \theta_{0}}{1+\frac{\sin \phi_{0}}{r_{0}}}$, and

$$
E_{\text {sno }}=\frac{\hat{\gamma}_{0}}{1+\zeta \frac{1}{R_{0}}} \text {. }
$$

The stress resultants are defined as (see Appendix D)

$$
N_{\text {so }}=\int_{-\frac{h}{2}}^{\frac{h}{2}} \sigma_{s o}\left(1+\zeta \frac{\sin \phi_{0}}{r_{0}}\right) d \zeta,
$$

$$
N_{\hat{N}_{0}}=\int_{-\frac{h}{2}}^{\frac{h}{2}} \sigma_{\theta_{0}}\left(1+\zeta \frac{1}{R_{0}}\right) d \zeta \text {, and }
$$

$$
Q_{0}=\int_{-\frac{h}{2}}^{\frac{h}{2}} \tau_{0}\left(1+\zeta \frac{\sin \phi_{0}}{r_{0}}\right) d \zeta .
$$


Note, $\frac{1}{R_{0}}=\frac{d_{\phi_{0}}}{d s_{0}}$.

Similarly the bending moments are defined as

$M_{\text {so }}=\int_{-\frac{h}{2}}^{\frac{h}{2}} \sigma_{\text {so }} \zeta\left(1+\zeta \frac{\sin \psi_{0}}{r_{0}}\right) d \zeta$, and

$M_{\theta_{0}}=\int_{-\frac{h}{2}}^{\frac{h}{2}} \sigma_{\theta_{0}} \zeta\left(I+\zeta \frac{1}{R_{0}}\right) d \zeta$.

Thus

$N_{s o}=C_{s} \hat{\varepsilon}_{s o}+C_{s A} \varepsilon_{\theta 0}+T_{s} \kappa_{s o}+T_{s \theta} \kappa_{\theta 0}$,

$N_{\theta 0}=C_{s \theta} \hat{\varepsilon}_{s o}+C_{\theta} \varepsilon_{\theta_{0}}+T_{s \theta} \kappa_{s o}+T_{\theta} \kappa_{\theta_{0}}$,

$Q_{0}=G \hat{\gamma}_{0}$,

$M_{s o}=T_{s} \hat{\varepsilon}_{s o}+T_{s \theta} E_{\theta_{0}}+D_{s} \kappa_{s o}+D_{s \theta} \kappa_{\theta 0}$, and

$M_{\theta 0}=T_{s \theta} \hat{\varepsilon}_{s o}+T_{\theta} \varepsilon_{\ni 0}+D_{s \theta} \kappa_{\text {so }}+D_{\theta} \kappa_{\theta_{0}}$,

where

$c_{s}=\int_{-\frac{h}{2}}^{\frac{h}{2}} \bar{c}_{s}\left(1+\zeta \frac{\sin \phi_{0}}{r_{0}}\right)\left(1+\zeta \frac{1}{R_{0}}\right)^{-1} d \zeta$, 


$$
\begin{aligned}
& c_{s \in}=\int_{-\frac{h}{2}}^{\frac{h}{2}} \bar{c}_{s \theta} d \zeta, \\
& c_{\theta}=\int_{-\frac{h}{2}}^{\frac{h}{2}} \bar{c}_{\theta}\left(1+\zeta \frac{1}{R_{0}}\right)\left(1+\zeta \cdot \frac{\sin \phi_{0}}{r_{0}}\right)^{-1} d \zeta, \\
& D_{s}=\int_{-\frac{h}{2}}^{\frac{h}{2}} \bar{c}_{s} \zeta^{2}\left(1+\zeta \frac{\sin \phi_{0}}{r_{0}}\right)\left(1+\zeta \frac{1}{R_{0}}\right)^{-1} d \zeta, \\
& D_{s \theta}=\int_{-\frac{h}{2}}^{\frac{h}{2}} \bar{c}_{s \theta^{\tau^{2}}}{ }^{2} d \zeta, \\
& D_{E}=\int_{-\frac{h}{2}}^{\frac{h}{2}} \bar{C}_{\theta} \zeta^{2}\left(1+\zeta \frac{1}{R_{0}}\right)\left(1+\zeta \frac{\sin \phi_{0}}{r_{0}}\right)^{-1} d \zeta, \\
& G=\int_{-\frac{h}{2}}^{\frac{h}{2}} \bar{G}\left(1+\zeta \frac{\sin \phi_{0}}{r_{0}}\right)\left(1+\zeta \frac{1}{R_{0}}\right)^{-1} d \zeta, \\
& T_{S}=\int_{-\frac{h}{2}}^{\frac{h}{2}} \zeta_{C_{S}}\left(1+\zeta \frac{\sin \phi}{r_{0}}\right)\left(1+\zeta \frac{1}{R_{0}}\right)^{-1} d \tau,
\end{aligned}
$$




$$
\begin{aligned}
& T_{s i}=\int_{-\frac{h}{2}}^{\frac{h}{2}} \bar{c}_{s \theta} \zeta d \zeta \text {, and } \\
& T_{e}=\int_{-\frac{h}{2}}^{\frac{h}{2}} \bar{c}_{\partial} \zeta\left(1+\zeta \frac{1}{R_{0}}\right)\left(1+\zeta \frac{\sin \phi_{0}}{r_{0}}\right)^{-1} d_{\zeta} .
\end{aligned}
$$

Note that

(1) for plastic material, $\overline{\mathrm{C}}_{s}, \overline{\mathrm{C}}_{\theta}$, and $\overline{\mathrm{C}}_{\mathrm{s} \theta}$ may vary with $\tau$, and

(2) for isotropic material,

$\bar{c}_{s}=\frac{E}{1-v^{2}}=\bar{c}_{\theta}, \quad$ and $\quad \bar{C}_{s \theta}=v \bar{c}_{s}$.

For thin shells

$\zeta \frac{\sin \phi_{0}}{r_{0}} \ll 1 \quad$ and $\quad \zeta \frac{1}{R_{0}} \ll 1$.

For thick shells

$\left(1+\zeta \frac{1}{R_{0}}\right)^{-1}=1-\zeta \frac{1}{R_{0}} \quad$ and $\left(1+\zeta \frac{\sin \phi_{0}}{r_{0}}\right)^{-1}=1-\zeta \frac{\sin \phi_{0}}{r_{0}}$.

The extension to thick shells has not yet been made in the computer code discussed in Ref. 8. 
In this appendix we will derive general strains in terms of the shell theory strains (membrane strains and bending strains). This exercise will demonstrate some of the limitations of this theory. Reference 1 discusses general strains for the small strain condition.

For this discussion we will need geometric relations for three separate states. These are illustrated with Figs. $\mathrm{C}-1--\mathrm{C}-3$. They are as follows.

(1) Undeformed state (Fig. C-1):

$$
\begin{aligned}
& \Delta s_{0} \Delta \xi=q_{0} \Delta \xi=R_{0} \Delta \phi_{0}, \\
& \Delta s_{0}(\zeta)=\left(1+\zeta \frac{1}{R_{0}}\right) \Delta s_{0}, \\
& \frac{\Delta r_{0}}{\Delta s_{0}}=\cos \phi_{0}, \\
& \frac{\Delta z_{0}}{\Delta s_{0}}=-\sin \phi_{0}, \\
& r_{0}(\zeta)=r_{0}+\zeta \sin \phi_{0}, \\
& z_{0}(\zeta)=z_{0}+\zeta \cos \phi_{0}, \\
& \Delta r_{0}(\zeta)=\Delta r_{0}+\zeta \Delta \phi_{0} \cos \phi_{0}, \text { and } \\
& \Delta z_{0}(\zeta)=\Delta z_{0}-\zeta \Delta \phi_{0} \sin \phi_{0},
\end{aligned}
$$

(2) Deformed state (Fig. C-2):

$$
\begin{aligned}
& \Delta s=q \Delta \xi=R(\Delta \phi-\Delta \gamma), \\
& \frac{\Delta r(\zeta)}{\Delta s(\zeta)}=\cos (\phi-\Gamma(\zeta)),
\end{aligned}
$$




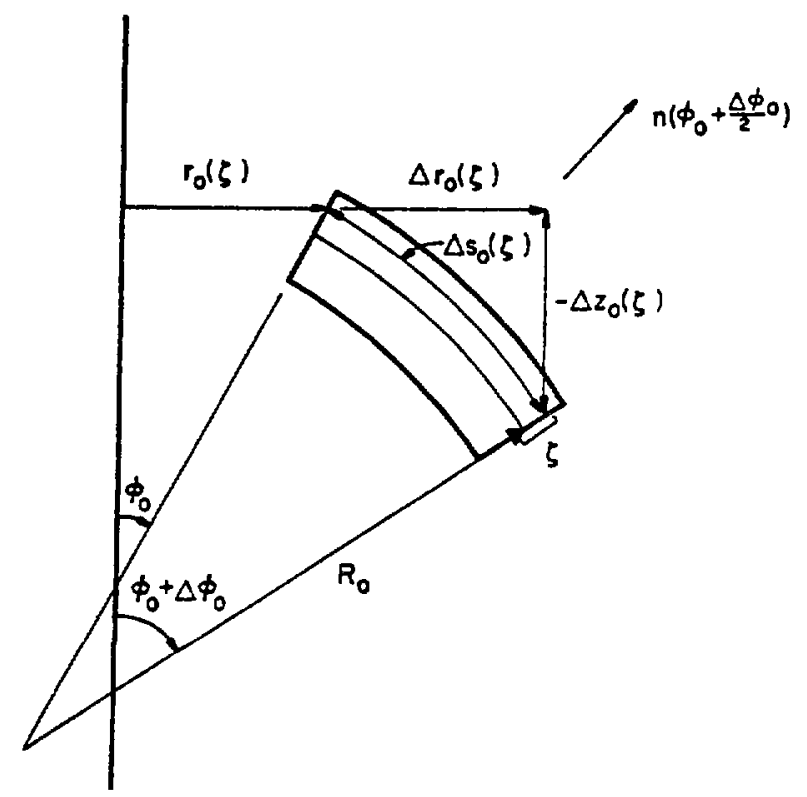

Fig. $C-1$.

Meridional cross-sectional view of incremental element (original geometry).

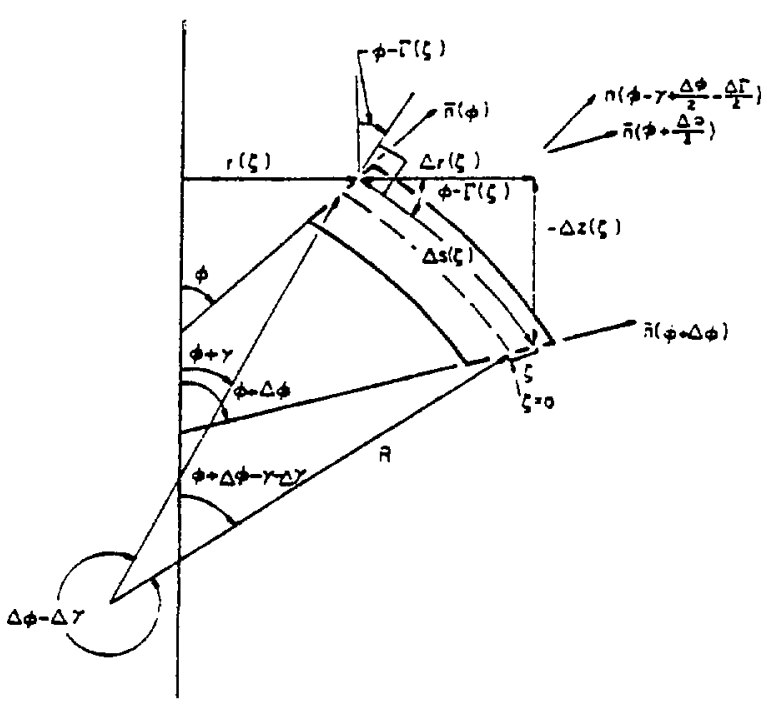

Fig. C-2.

Meridional cross-sectional view of incremental element (deformed geometry).

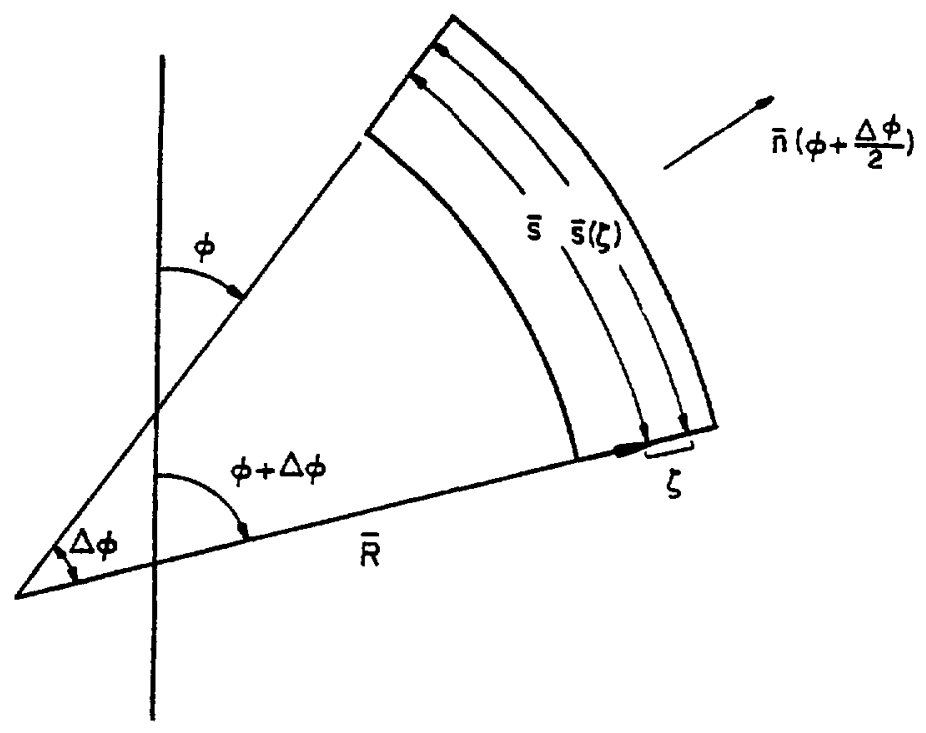

Fig. C-3.

Meridional cross-sectional view of incremental element (deformed geometry except for shear deformation). 


$$
\begin{aligned}
& \frac{\Delta z(\zeta)}{\Delta s(\zeta)}=-\sin (\phi-\Gamma(\zeta)), \\
& \frac{\Delta r}{\Delta s}=\cos (\phi-\gamma), \\
& \frac{\Delta z}{\Delta s}=-\sin (\phi-\gamma), \\
& r(\zeta)=r+\zeta \sin \phi, \\
& z(\zeta)=z+\zeta \cos \phi, \\
& \Delta r(\zeta)=\Delta r+\zeta \Delta \phi \cos \phi, \text { and } \\
& \Delta z(\zeta)=\Delta z-\zeta \Delta \phi \sin \phi .
\end{aligned}
$$

(3) Deformed state without shear deformation (Fig. C-3):

$$
\begin{aligned}
& \Delta \overline{\mathrm{S}}=\bar{q} \Delta \xi=\overline{\mathrm{R}} \Delta \phi \quad \text { and } \\
& \Delta \overline{\mathrm{s}}(\zeta)=\left(1+\zeta \frac{1}{\bar{R}}\right) \Delta \overline{\mathrm{s}}=\left(1+\zeta \frac{\Delta \phi}{\Delta \overline{\mathrm{s}}}\right) \Delta \overline{\mathrm{s}}
\end{aligned}
$$

From Figs. $\mathrm{C}-2$ and $\mathrm{C}-3$ we can see that.

$\Delta \bar{s}=\Delta s \cos \gamma$ and $\Delta \bar{s}(\zeta)=\Delta s(\zeta) \cos \Gamma(\zeta)$.

Bec ause

$$
\Delta \overline{\mathbf{s}}(\zeta)=\left(1+\zeta \frac{\Delta \phi}{\Delta \overline{\mathbf{s}}}\right) \Delta \overline{\mathbf{s}}, \quad \Delta \mathrm{s}(\zeta)=\frac{1}{\cos \Gamma(\zeta)}\left(\cos \gamma+\zeta \frac{\Delta \phi}{\Delta \mathbf{s}}\right) \Delta \mathbf{s} .
$$

Consider the following strains with respect to the deformed configuration. 


$$
\begin{aligned}
& E_{\theta}=\frac{r(\zeta)-r_{0}(\zeta)}{r(\zeta)}, \\
& E_{s}=\frac{\Delta \bar{s}(\zeta)-\Delta s_{0}(\zeta)}{\Delta \bar{s}(\zeta)}, \text { and } \\
& E_{s n}=\tan \Gamma(\zeta) .
\end{aligned}
$$

From Eqs. $(C-1)$ and $(C-2), r(\zeta)=r+\zeta \sin \phi$ and $r_{0}(\zeta)=r_{0}+\zeta \sin \phi_{0}$, the hoop strain is

$E_{\theta}=\frac{\left(\frac{r-r_{0}}{r}\right)+\zeta\left(\frac{\sin \phi-\sin \phi_{0}}{r}\right)}{1+\zeta \frac{\sin \phi}{r}}=\frac{\varepsilon_{\theta}+\zeta \kappa_{\theta}}{1+\zeta \frac{\sin \phi}{r}}$.

The meridional strain $E_{S}$ can be written as (from Eq. (C-6))

$$
E_{s}=1-\frac{\Delta s_{0}(\zeta)}{\Delta \bar{s}(\zeta)} \text {. }
$$

From Eqs. $(\mathrm{C}-1),(\mathrm{C}-4)$, and $(\mathrm{C}-5)$, the meridional strain $(\mathrm{C}-8)$ can be written as

$$
E_{s}=1-\left(\frac{\frac{q_{0}}{q}+\zeta \frac{\Delta \phi_{0}}{\Delta s}}{\cos \gamma-\zeta \frac{\Delta \phi}{\Delta s}}\right)
$$

or

$E_{S}=\frac{\cos \gamma-\frac{q_{0}}{q}+\zeta\left(\frac{\Delta \phi}{\Delta s} \cdot \frac{\Delta \phi_{0}}{\Delta s}\right)}{\cos \gamma-\zeta \frac{\Delta \phi}{\Delta s}}=\frac{1-\frac{q_{0}}{q \cos \gamma}+\zeta\left(\frac{\Delta \phi}{\Delta \bar{s}}-\frac{\Delta \phi_{0}}{\Delta \bar{s}}\right)}{1-\zeta \frac{1}{\bar{R}}}=\frac{\hat{\varepsilon}_{s}+\zeta k}{1+\zeta \frac{1}{\bar{R}}}$. 
Next, we will determine a relation for the shearing strain $E_{s n}$. From the following (C-2) equations,

$$
\begin{aligned}
& \Delta r(\zeta)=\Delta s(\zeta) \cos (\phi-\Gamma(\zeta)), \\
& \Delta z(\zeta)=-\Delta s(\zeta) \sin (\phi-\Gamma(\zeta)), \\
& \Delta r \quad=\Delta s \cos (\phi-\gamma), \text { and } \\
& \Delta z=-\Delta s \sin (\phi-\gamma),
\end{aligned}
$$

the following equation can be derived

$$
\sin \Gamma(\zeta)=\frac{\Delta s}{\Delta s(\zeta)} \sin \gamma
$$

Substituting Eq. (C-5) into Eq. (C-12) gives

$$
E_{\text {Sn }}=\tan \Gamma(\zeta)=\frac{\sin \gamma}{\cos \gamma+\zeta \frac{\Delta \phi}{\Delta s}}=\frac{\tan \gamma}{1+\zeta \frac{\Delta \phi}{\cos \gamma \Delta s}} .
$$

From Eq. $(C-3), \Delta \bar{s}=\cos \gamma \Delta s, \quad$ and from Eq. $(C-3), \quad \frac{1}{\bar{R}}=\frac{\Delta \phi}{\Delta \vec{S}} \cdot$

Thus Eq. $(C-13)$ can be written as

$$
E_{s n}=\frac{\tan \gamma}{1+\zeta \frac{\bar{l}}{\bar{R}}}=\frac{\hat{P}}{1+\zeta \frac{\overline{1}}{\bar{R}}}
$$

Consider the following strains with respect to the undeformed configuration.

$$
E_{\theta 0}=\frac{r(\zeta)-r_{0}(\zeta)}{r_{0}(\zeta)} \text { and } E_{s 0}=\frac{\overline{\Delta s}(\zeta)-\Delta s_{0}(\zeta)}{\Delta s_{0}(\zeta)} \text {. }
$$


From Eqs. (C-1) and (C-2), $r(\zeta)=r+\zeta \sin \phi$ and $r_{0}(\zeta)=r_{0}+\zeta \sin \phi_{0}$, the hoop strain is

$$
E_{\theta_{0}}=\frac{\left(\frac{r-r_{0}}{r_{0}}\right)+\zeta\left(\frac{\sin \phi-\sin \phi_{0}}{r_{0}}\right)}{1+\zeta \frac{\sin \phi_{0}}{r_{0}}}=\frac{\varepsilon_{\theta_{0}}+\zeta \kappa_{\theta_{0}}}{1+\zeta \frac{\sin \phi_{0}}{r_{0}}} .
$$

Next, we will consider the meridional strain $E_{\text {so }}$. Substituting an equation from Eq. (C-4) into the equation for $E_{\text {so }}$ in Eq. (C-15),

$\frac{\Delta s(\zeta)}{\Delta s_{0}(\zeta)} \cos \Gamma(\zeta)=1+E_{s 0}$, and thus

$\Delta s(\zeta)=\frac{\left(1+E_{S O}\right)}{\cos \Gamma(\zeta)} \Delta s_{0}(\zeta)=\frac{\left(1+E_{S O}\right)}{\cos \Gamma(\zeta)}\left(1+\zeta \frac{1}{R_{0}}\right) \Delta s_{0}$.

A 1so, from Eqs. (C-5), (C-1), and $(C-2)$,

$$
\Delta s(\zeta)=\frac{1}{\cos \Gamma(\zeta)}\left(\cos \gamma+\zeta \frac{\Delta \phi}{\Delta s}\right) \Delta s \quad \text { and } \quad \frac{\Delta s}{\Delta s}=\frac{q}{q_{0}} .
$$

Thus

$$
\Delta s(\zeta)=\frac{1}{\cos \Gamma(\zeta)}\left(\cos \gamma+\zeta \frac{\Delta \phi}{\Delta s}\right) \frac{q}{q_{0}} \Delta s_{0} .
$$

From Eqs. (C-17) and (C-19),

$$
\left(1+E_{\text {so }}\right)\left(1+\zeta \frac{1}{R_{0}}\right)=\left(\cos \gamma+\zeta \frac{\Delta \phi}{\Delta s}\right) \frac{q}{q_{0}} ;
$$

thus $E_{s 0}=\frac{\frac{q}{q_{0}} \cos \gamma-1+\zeta\left(\frac{\Delta \phi}{\Delta s_{0}}-\frac{\Delta \phi_{0}}{\Delta s_{0}}\right)}{1+\zeta \frac{1}{R_{0}}}=\frac{\hat{\varepsilon}_{s 0}+\zeta \kappa_{s 0}}{1+\zeta \frac{1}{R_{0}}}$. 
Last, we will determine a relation for the shearing strain $E_{\text {sno }}$. From Eqs. $(C-7),(C-10),(C-16)$, and $(C-20)$,

$$
\varepsilon_{\theta_{0}}=\frac{r}{r_{0}} \varepsilon_{\theta},
$$$$
\hat{\varepsilon}_{s o}=\frac{q \cos \gamma}{q_{0}} \hat{\varepsilon}_{s},
$$$$
k_{\text {so }}=\frac{q \cos Y}{q_{0}} r_{s} \text {, and }
$$

$$
\kappa_{\theta 0}=\frac{r}{r_{0}} \kappa_{\theta} .
$$

Also, from Ref. 2,

$$
\hat{\gamma}_{0}=\frac{q}{q_{0}} \sin \gamma
$$

Thus, from Eq. $(C-14)$ we can show

$$
\hat{\gamma}_{0}=\frac{q \cos \gamma}{q_{0}} \hat{\gamma}
$$

An inspection of Eqs. (C-20) and (C-14) shows that

$$
E_{\text {sno }}=\frac{\hat{\gamma}_{0}}{1+\zeta \frac{1}{R_{0}}} .
$$




\section{APPENDIX D \\ STRESS RESULTANTS AND BENDING MOMENTS}

The stress resultants and bending moments as shown in Fig. A-1 are defined as

$$
\begin{aligned}
& N_{s}=\int_{-\frac{h}{2}}^{\frac{h}{2}} \sigma_{\bar{s}}\left(1+\zeta \frac{\sin \phi}{r}\right) d \zeta, \\
& N_{\theta}=\int_{-\frac{h}{2}}^{\frac{h}{2}} \sigma_{\theta}\left(1+\zeta \frac{1}{\bar{R}}\right) d \zeta, \\
& Q=\int_{-\frac{h}{2}}^{\frac{h}{2}} \tau\left(1+\zeta \frac{\sin \phi}{r}\right) d \zeta, \\
& M_{s}=\int_{-\frac{h}{2}}^{\frac{h}{2}} \sigma_{\bar{s}} \zeta\left(1+\zeta \frac{\sin \phi}{r}\right) d \zeta \text {, and } \\
& M_{\theta}=\int_{-\frac{h}{2}}^{\frac{h}{2}} \sigma_{\theta} \zeta\left(1+\zeta \frac{1}{\bar{R}}\right) d \zeta,
\end{aligned}
$$

where 
$\cos \Gamma(\zeta) \doteq \cos \gamma$ in Eq. $(C-5)$, and

$\frac{1}{\bar{R}}=\frac{d \phi}{d \bar{s}}$, as shown in Fig. C-3.

The stresses $\sigma_{\dot{s}}, \sigma_{\theta}$, and $\tau$ are Cauchy Stresses (also called Eulerian or true stresses, see Ref. 9).

From Eq. (2)

$N_{s o}=\frac{r}{r_{0}} N_{s}, Q_{0}=\frac{r}{r_{0}} Q, M_{s o}=\frac{r}{r_{0}} M_{s}, N_{\theta 0}=\frac{q}{q_{0}} N_{\theta}$, and $H_{e_{0}}-\frac{a}{q_{0}} M_{\theta 0}$.

Let the Lagrangian strains $\sigma_{s 0}, \sigma_{\theta 0}$, and $\tau_{0}$ be defined as (see Ref. 9)

$$
\begin{aligned}
& \sigma_{\text {so }}\left(r_{0}+\zeta \sin \phi_{0}\right)=\sigma_{\bar{s}}(r+\zeta \sin \phi), \\
& \sigma_{\theta 0} q_{0}\left(1+\frac{\zeta}{R_{0}}\right)=\sigma_{\theta} q\left(1+\frac{\zeta}{\bar{R}}\right), \text { and } \\
& \tau_{0}\left(r_{0}+\zeta \sin \phi_{0}\right)=\tau(r+\zeta \sin \phi) .
\end{aligned}
$$

When Eq. $(D-3)$ is substituted into Eq. (D-1) and Eq. (D-1) is substituted into Eq. $(D-2)$, Eqs. $(B-3)$, and $(B-4)$,

$$
N_{\text {so }}=\int_{-\frac{h}{2}}^{\frac{h}{2}} \sigma_{s o}\left(1+\zeta \frac{\sin \phi_{0}}{r_{0}}\right) d \zeta,
$$




$$
\begin{aligned}
& N_{\theta 0}=\int_{-\frac{h}{2}}^{\frac{h}{2}} \sigma_{\theta 0}\left(01+\zeta \frac{1}{R_{0}}\right) d \zeta, \\
& Q_{0}=\int_{-\frac{h}{2}}^{\frac{h}{2}} \tau_{0}\left(1+\zeta \frac{\sin \phi_{0}}{r_{0}}\right) d \zeta . \\
& M_{s 0}=\int_{-\frac{h}{2}}^{\frac{h}{2}} \sigma_{s 0} \zeta\left(1+\zeta \frac{\sin \phi_{0}}{r_{0}}\right) d \zeta, \text { and } \\
& M_{\theta 0}=\int_{-\frac{h}{2}}^{\frac{h}{2}} \sigma_{\theta 0} \zeta\left(1+\zeta \frac{1}{R_{0}}\right) d \zeta .
\end{aligned}
$$

The thickness $h$ is the thickness in the deformed configuration,

\section{APPENDIX E \\ NONLINEAR GEOMETR IC PROBLEMS}

Axially Loaded Cylinder

Consider the cylinder shown in Fig. 7. What are the displacements for an axially loaded cylinder? From the equilibrium equation Eq. (5), 
$\frac{d\left(r_{0} N_{s 0}\right)}{d s_{0}}=0, N_{e_{0}}=0, Q_{0}=0, M_{s o}=0, M_{\theta 0}=0$, and $P_{0}=0$.

Also, there is no shear deformation. Thus

$r_{0} N_{\text {so }}=$ constant.

From Eq. (2) of Sec. II,

$r_{0} N_{\text {so }}=r N_{S}=F$, or $\quad N_{\text {so }}=\frac{F}{r_{0}} \quad(\Delta \theta=1$ radian $)$.

The constitutive equations for these stress resultants give

$N_{\text {so }}=\frac{F}{r_{0}}=C_{s} \varepsilon_{s o}+C_{s} \varepsilon_{\theta_{0}}$, and

$N_{\theta 0}=0=C_{\theta} \varepsilon_{\text {so }}+C_{\theta} \varepsilon_{\theta 0}$,

where $\varepsilon_{0}=\frac{q}{q_{0}}-1$, and $\varepsilon_{\theta 0}=\frac{u_{r}}{r_{0}}$.

Solving these equations for $\varepsilon_{\text {so }}$ and $\varepsilon_{\theta_{0}}$ gives

$\varepsilon_{\theta 0}=\frac{-c_{s \theta} F}{r_{0}\left(c_{s} c_{\theta}-c_{s \theta}^{2}\right)}$,
$\varepsilon_{s o}=\frac{c_{\theta} F}{r_{0}\left(c_{s} c_{\theta}-c_{s \theta}^{2}\right)}$. 
Because $\frac{q}{q_{0}}=\frac{2}{\ell_{0}}$,

$u_{r}$ and $\ell$ can be solved for as

$$
u_{r}=\frac{-c_{s \theta} F}{c_{s} c_{\theta}-c_{s \theta}^{2}} \quad \text { and } \quad l=\frac{\ell_{0} c_{\theta} F}{r_{0}\left(c_{s} c_{\theta}-c_{s \theta}^{2}\right)}+l_{0} \text {. }
$$

$$
\text { For } \begin{aligned}
F & =10^{6} \mathrm{lb}, \\
r_{0} & =10 \mathrm{in.}, \\
\ell_{0} & =5 \mathrm{in.}, \\
E & =10^{6} \mathrm{psi}, \\
\nu & =0.25,
\end{aligned}
$$

and $\mathrm{h}=0.1$, the materials are

$c_{s}=C_{\theta}=\frac{E h}{1-v^{2}}=1.0666710^{5} \mathrm{lb} / \mathrm{in}$. , and

$C_{S \theta}=\frac{v E h}{1-v^{2}}=.26666710^{5} \mathrm{lb} / \mathrm{in}$.

Thus

$u_{r}=-2.5 \mathrm{in.} \quad$ and $\quad \ell=10 \mathrm{in}$.

Expansion of a Hemisphere

Consider the hemisphere in Fig. 8. What pressure is required to double the size of the henisphere? From Fig. $8, u_{s}=0, u_{n}=c$, and $\phi=\phi_{0}$. Thus the membrane and bending strains are

$$
\varepsilon_{\text {so }}=\frac{q}{q_{0}}-1=\frac{a+c}{a}-1=\frac{c}{a}, \quad \varepsilon_{\theta_{0}}=\frac{c \sin \phi_{0}}{a \sin \phi_{0}}=\frac{c}{a} \text {, }
$$


$\gamma=0$ (no shear deformation), $\kappa_{\text {so }}=0, \kappa_{\theta 0}=0$, and $P_{0}=0$.

The constitutive equations for this membrane problem of isotropic material $c$ an be written as (includes thermal strains)

$N_{\text {so }}=\frac{E h}{1-\nu^{2}} \quad\left\{\varepsilon_{\text {so }}-\alpha \Delta+\nu\left(\varepsilon_{\theta_{0}}-\alpha \Delta\right)\right\}$, and

$(E-9)$

$N_{\partial 0}=\frac{E h}{1-\nu^{2}}\left\{\varepsilon_{\theta 0}-\alpha \Delta+\nu\left(\varepsilon_{\text {so }}-\alpha \Delta\right)\right\}$.

Because $\varepsilon_{\text {so }}=\varepsilon_{\theta 0}$,

$N_{\text {so }}=N_{\theta_{0}}=\frac{E h}{1-\nu}(c / a-\alpha \Lambda)$.

Aga in from Fig. 8

$r_{0}=a \sin \phi_{0}$ and $R_{0}=a$,

$(E-10)$

and thus the equilibrium equation, Eq. (5), gives

$-\frac{r_{0}}{R_{0}} N_{s o}-N_{0} \sin \phi_{0}+r_{0} p_{0}=0$,

$(E-11)$

which results in

$p_{0}=\frac{N_{S O}+N_{E O}}{a}=\frac{2 E h}{a(1-\nu)}(c / a-\alpha \Delta)$.

$(E-12)$

A iso $p=p_{0} \frac{r_{0} q_{0}}{r q}=p_{0} \frac{a^{2}}{(a+c)^{2}}$

(E-13)

41 


$$
\text { for } \begin{aligned}
a & =12.0 \mathrm{in.}, \\
c & =12.0 \mathrm{in.}, \\
E & =10^{6} \mathrm{psi}, \\
\nu & =.25, \\
h & =.4 \mathrm{in.}, \text { and } \\
\alpha \Delta & =.25(\text { thermal strain), the loads are } \\
p_{0} & =.66667 \times 10^{5} \mathrm{psi}, \text { and } \\
p & =.16667 \times 10^{5} \mathrm{psi} .
\end{aligned}
$$

\section{Cylinder Deforms Into a Spherical Shape}

For this problem we will assume a cylinder deforms into a spherical shape, and then we calculate the loads that would be necessary to do this. Thus from Fig. 9

$$
\begin{aligned}
& \phi_{0}=\pi / 2, r=a \sin (\phi-\gamma), z=a \cos (\phi-\gamma), \\
& s=a(\phi-\gamma)-a\left(\phi_{i}-\gamma_{i}\right), \text { and } \phi=\frac{s}{\alpha}+\gamma+\left(\phi_{i}-\gamma_{i}\right) ;
\end{aligned}
$$

also $\frac{q}{q_{0}}=\frac{a\left(\pi / 2-\phi_{i}+\gamma_{i}\right)}{l_{0}}$ (a constant).

Thus the membrane strains

$$
\hat{\varepsilon}_{\text {so }}=\frac{q}{q_{0}} \cos \gamma-1, \varepsilon_{\theta_{0}}=\frac{a \sin (\phi-\gamma)}{r_{0}}-1, \text { and } \gamma_{0}=\frac{q}{q_{0}} \sin \gamma \text {. }
$$

The bending strains are

$$
\kappa_{\text {so }}=\frac{d B}{d s_{0}}=\frac{q}{q_{0}} \frac{d B}{d s}=\frac{q}{q_{0}}\left(\frac{d \phi}{d s}\right)=\frac{q}{q_{0}}\left(\frac{1}{a}+\frac{d \gamma}{d s}\right) \text {, }
$$

where $\beta=\phi-\phi_{0}, k_{\theta_{0}}=\frac{\sin \phi}{r_{0}}-\frac{1}{r_{0}}$, and $\lambda_{0}=\frac{\cos \phi}{r_{0}}$. 
For an isotropic material (except for shear modulus $G$ ), the stress resultants are

$$
\begin{aligned}
& N_{s o}=C\left\{\frac{q}{q_{0}} \cos \gamma+\frac{v a}{r_{0}} \sin (\phi-\gamma)-(1+v)(1+\alpha \Delta)\right\}, \\
& N_{\theta_{0}}=C\left\{\frac{v q}{q_{0}} \cos \gamma+\frac{a}{r_{0}} \sin (\phi-\gamma)-(1+\nu)(1+\alpha \Delta)\right\}, \text { and } \\
& Q_{0}=\bar{G} \frac{q}{q_{0}} \sin \gamma,
\end{aligned}
$$

where

$$
C=\frac{-E h}{1-v^{2}} \quad \text { and } \quad \bar{G}=\text { Gr } .
$$

The bending moments are

$$
\begin{aligned}
& M_{s o}=D\left\{\frac{q}{q_{0}}\left(\frac{1}{a}+\frac{d y}{d s}\right)+v\left(\frac{\sin \phi}{r_{0}}-\frac{1}{r_{0}}\right)\right\}, \\
& M_{\theta_{0}}=D\left\{\frac{v q}{q_{0}}\left(\frac{1}{a}+\frac{d y}{d s}\right)+\frac{\sin \phi}{r_{0}}-\frac{1}{r_{0}}\right\}, \\
& P_{0}=D \frac{\cos \phi}{r_{0}},
\end{aligned}
$$

where

$$
D=\frac{E h^{3}}{12\left(1-v^{2}\right)} \text {. }
$$

43 
When these quantities are substituted into the equilibrium equations, Eq. (5), the rotational equation gives

$B_{1} \frac{d^{2} \gamma}{d s^{2}}+B_{2} \gamma+B_{3}=0$

where

$B_{1}=D r_{0}\left(\frac{q}{q_{0}}\right)^{2}$,

$B_{2}=(C-\bar{G}) r_{0}\left(\frac{q}{q_{0}}\right)^{2}-C r_{0} \frac{q}{q_{0}}(1+v)(1+\alpha \Delta)+v C a\left(\frac{q}{q_{0}}\right) \sin \left(\frac{s}{a}+\phi_{i}-\gamma_{i}\right)$

$-\frac{D \lambda}{r_{0}} \sin \left(\frac{s}{a}+\phi_{i}-\gamma_{i}\right)$,

$B_{3}=\frac{D \lambda}{r_{0}} \cos \left(\frac{s}{a}+\phi_{i}-\gamma_{i}\right), \quad$ and

$\gamma$ is as sumed small.

The boundary conditions for this equation are

(1) $\gamma=0$ at $\phi=\pi / 2$, and

(2) $\frac{d \gamma}{d s}=0$ at $\phi=\pi / 2$ (symetry boundary condition).

Equation (E-19) can be solved using a finite difference scheme as follows.

$B_{1}\left(\frac{\gamma_{i+1}-2 \gamma_{i}+\gamma_{i-1}}{(\Delta s)^{2}}\right)+\gamma_{i} B_{2}+B_{3}=0$ 
where $[i]$ is a counter for the incremental.

$\gamma_{i+1}=2 \gamma_{i}-\gamma_{i-1}-\frac{(\Delta s)^{2}}{B_{1}}\left(\gamma_{i} B_{2}+B_{3}\right)$ or, if $\left|B_{1} \frac{d^{2} \gamma}{d s^{2}}\right| \ll B_{1} \gamma+B_{3}$,

then $\gamma_{i} B_{2}-B_{3}=0$, and

$\gamma_{i}=\frac{B_{3}}{B_{2}}$

With the $\gamma$ known, the two equilibrium equations, Eq. (6), give the loads required to deform a cylinder into a spherical shape. These are

$p_{H_{0}}=\sin \left(\frac{q}{q_{0}}\right)^{2}\left\{\frac{C}{a}+(C-\bar{G}) \frac{d \gamma}{d s}\right\}+\gamma \cos \left(\frac{q}{q_{0}}\right)^{2}\left\{-\frac{\bar{G}}{a}+(C-\bar{G}) \frac{d \gamma}{d s}\right\}$

$-\sin \phi\left(\frac{q}{q_{0}}\right) C(1+\nu)\left\{\frac{1}{a}+\frac{d \gamma}{d s}\right\}-\frac{\nu C}{r_{0}}\left(\frac{q}{q_{0}}\right) \cos (\phi-\gamma) \cos \phi$

$+\frac{\nu C}{r_{0}}\left(\frac{q}{q_{0}}\right) a \sin (\phi-\gamma) \sin \phi\left\{\frac{1}{a}+\frac{d \gamma}{d s}\right\}+\frac{\nu C}{r_{0}}\left(\frac{q}{q_{0}}\right) \cos \gamma+\frac{C a}{r_{0}^{2}} \sin (\phi-\gamma)$

$-(1-v) \frac{C}{r_{0}}-\left(\frac{q}{q_{0}}\right) \sin \phi\left\{\frac{1}{a}+\frac{d \gamma}{d s}\right\}(1+v) \alpha \Delta C-\frac{C}{r_{0}}(1+v) \alpha \Delta$

and

$p_{v_{0}}=\cos \phi\left(\frac{q}{q_{0}}\right)^{2}\left\{\frac{C}{d}+(C-\bar{G}) \frac{d y}{d s}\right\}+\gamma \sin \phi\left(\frac{q}{q_{0}}\right)^{2}\left\{\frac{\bar{G}}{a}+(\bar{G}-C) \frac{d \gamma}{d s}\right\}$

$-\cos \phi\left(\frac{q}{q_{0}}\right) c(1+\nu)\left\{\frac{1}{a}+\frac{d y}{d s}\right\}+\frac{\nu C}{r_{0}}\left(\frac{q}{q_{0}}\right) \cos (\phi-\gamma) \sin \phi$ 
$+\frac{\nu C a}{r_{0}}\left(\frac{q}{q_{0}}\right) \sin (\phi-\gamma) \cos \phi\left\{\frac{1}{a}+\frac{d \gamma}{d s}\right\}+\cos (1+\nu)\left(\frac{q}{q_{0}}\right) \cos \phi\left\{\frac{1}{a}+\frac{d \gamma}{d s}\right\}$.

The problem solved used

$$
\begin{aligned}
& a=5, \\
& \ell_{0}=4, \\
& \alpha \Delta=0 \text { (thermal strain), } \\
& \nu=.25, \\
& E=10^{5}, \text { and } \\
& G=40 \mathrm{E} .
\end{aligned}
$$

\section{REFERENCES}

1. E. Reissner, "On Finite Symmetrical Deflections of Thin Shells of Revolution," J. Appl. Mechanics 36, Trans. ASME, Series E, 267-270 (1969).

2. E. Reissner, "On Finite Symmetrical Deflections of Thin Shelis of Revolution," J. App1. Mechanics 39, Trans. ASME, Series E, 1137-1138 (1972).

3. K. J. Bathe, E. L. Wi lson, and R. H. Iding, "NONSAP - A Structural Analys is Program for Static and Dynamic Response of Linear Systems," Dept. of Civil Eng., Univ. of California report UCSESM 74-3 (1974).

4. K. J. Bathe, H. Ozdemir, and E. L. Wilson, "Static and Dynamic Geometric and Material Nonlinear Analys is," Dept of Civil Eng., Univ. of California report UCSESM 74-4 (1974).

5. P. E. Grafton and P. R. Strome, "Analys is of Axisymetric Shells by the Direct Stiffness Method," AIAA J. 1, (10), 2342-2347 (1963). 
6. W. A. Cook, "Finite Element Linear Models for Symetrically Luadeu Shel ls of Revolution," Proceedings of 5 th International Semiriar on: Computational Aspects of Finite Element Methods, August 21-22, 1979, Berlin (West), Germany.

7. K. J. Bathe and A. P. Cimento, "Some Practical Procedures for the Solution of Non 1 inear Finite Element Equations," J. Computer Meth. in App 1. Mech. and Eng., in press.

8. T. A. Butler, E. G. Endebrock, and J. B. Payne, "CRASHC - A Two-Dimensional Code to Compute the Response of Axisymmetric Shipping Containers to End-onImpacts," Los Alamos Scientific Laboratory report to be published.

9. Y. C. Fung, Foundations of Solid Mechanics, Prentice Hal1, Inc., Englewood Cliffs, New Jersey (1965). 